\title{
TABLE CAPE VENT XENOLITH SUITE, NORTHWEST TASMANIA: MINERALOGY AND IMPLICATIONS FOR CRUST-MANTLE LITHOLOGY AND MIOCENE GEO- THERMS IN TASMANIA
}

\author{
by F. L. Sutherland, L. R. Raynor and R. E. Pogson
}

(with one plate, two text-figures and eight tables)

\begin{abstract}
Sutherland, F. L., Raynor, L. R. \& Pogson, R. E. 2005 (16:xii): Table Cape vent xenolith suite, northwest Tasmania: Mineralogy and implications for crust-mantle lithology and Miocene geotherms in Tasmania. Papers and Proceedings of the Royal Society of Tasmania 139: 7-22. https://doi.org/10.26749/rstpp.139.7 ISSN 0080-4703. Mineralogy \& Petrology, Australian Museum, 6 College Street, Sydney, New South Wales 2010 Australia (FLS*, REP); deceased (LRR). *Author for correspondence.
\end{abstract}

\begin{abstract}
The Miocene Table Cape vent erupted a diverse mantle-crust xenolith suite within its fractionated nephelinitic matrix. Assemblages include mantle metaperidotites, garnet-metawebsterites and rarer garnet-metaclinopyroxenites, garnet-metawehrlites, metawebstetites and crustal two-pyroxene granulites. Most metapyroxenites and granulites represent the $\Gamma \mathrm{i}$-Al-bearing augite suite and their bulk geochemistry indicates transitional olivine basalt magmatic affinities. Metasomatised, hydrous lithologies are only rarely present. Co-existing pyroxenes in the xenoliths provide re-equilibration temperature estimates from $860-1075{ }^{\circ} \mathrm{C}$ (for the whole suite) and temperature-pressure cstimates for the garnet metawebsterites from $1055-1070^{\circ} \mathrm{C}$ and $1.2-1.4$ GPa. This gives a Miocene mantle geotherm gradient at least $80-130^{\circ} \mathrm{C}$ higher than the Southeast Australian (SEA) western Victorian geotherms. However, considerations of Moho depths from new seismic survcys below Table Cape $(-32 \mathrm{~km})$ suggest that the indicated geotherm is more strongly perturbed in its lower levels than at the mantle-crust transition. This localised perturbation is attributed to adjacent magna chamber activity in the mantle (Boat Harbour basalts) just prior to Table Cape vent activity. Tasmanian Miocene geotherms (Table Cape, Bow Hill) achieve relatively high gradients and reinforce suggestions of local variation in East Australian geothermal gradients. They illustrate the potential complexities in comparing xenolith-derived geotherms from different areas in general, both from thermometer/baroneter selection and from associated magmatic heat inputs.
\end{abstract}

Key Words: Table Cape volcano, Cenozoic, xenoliths, mineralogy, mantle, crust, thermobarometry, geotherms, magma chamber.

\section{INTRODUCTION}

Table Cape is a prominent bluff of coarse-grained alkali dolerite (although previously mapped as crinanite, the preferred specific nomenclature is now teschenite; see Le Maitre 1989). It forms a massive core of a substantially eroded volcanic centre (fig. 1). The teschenite fronts on to Bass Strait in cliffs up to $179 \mathrm{~m}$ a.s.l. (Wynyard 1:25000 Sheet; 55GCQ930655, AGC66, Everard 2003). This slightly differentiated body only becomes more distinctly alkaline in its chemistry in late-stage pegmatoidal veins (schlieren) and it incorporates ultramafic xenoliths and xenocrysts of mantle origin, particularly in its lower levels (Gee 1971, Piestrzeniewicz 1972, Everard 2001). Lavas from the Table Cape centre form a remnant apron, in parts deeply weathered, that was dated at $13.3 \pm 0.2 \mathrm{Ma}$ at Tollymore Road quarry (Sutherland \& Wellman 1986). The lavas overlie a range of older lithologies (fig. 1) and extend west of Table Cape to Jacobs Boat Harbour as a mappable unit of normal magnetic polarity (Sutherland et al. 2004, fig. 12).

Vent pyroclastic deposits up to $5 \mathrm{~m}$ thick outcrop on the south shore of Table Cape (55GCQ930648, AGD66). Here they underlie a north-dipping base to the teschenite and contain a range of mantle and lower crustal fragments within a basaltic and comminuted country rock matrix (Sutherland et al. 1989, Everard 2001, Sutherland et al. 2004, figs 8d-f, 10f). Rare fragments of Lower Miocene marine sediments in the pyroclastic beds indicate an age younger than the marine beds. The pyroclastics include irregular sill-like bodies of chilled tachylytic basalt that form a more resistant protection to the included fragments. The deeper origin xenoliths are important in reconstructing the underlying Tasmanian lithosphere and its geothermal state at the time of volcanism, as they not only include garnet-two pyroxene assemblages suitable for geothermobarometry but also diverse mantle peridotites and pyroxenites (Sutherland et al. 1994, 2004, Beyer 1998, Beyer et al. 2001). The Table Cape suite is one of only two known garnetiferous mantle xenolith sites in Tasmania, the other being at Bow Hill, west of Oatlands in the central midlands (fig. 1).

This study presents a more complete account of the Table Cape vent xenolith mineralogy and uses new thermobarometric methods to discuss their context within recent studies on the Southeast Australian lithosphere in the Cenozoic and its geothermal relationships (Graham et al. 2003, Sutherland et al. 2004, Roach 2004). The paper integrates the Table Cape vent xenolith data with Boat Harbour (Sutherland et al. 1996) and provides a revised lithospheric framework for the Wynyard-Boat Harbour region. The proposed lithology is then correlated with new seismic data on crust-mantle structures under northern Tasmania (Rawlinson et al. 2001, 2004).

\section{SAMPLES AND ANALYTICAL METHODS}

The Table Cape vent rocks contain numerous xenolithic and xenocrystic fragments. The fragments of mantle-lower crust origin include common spinel-metaperidotites up to $10 \mathrm{~cm}$, sparse garnet-metapyroxenites and granulites up to $8 \mathrm{~cm}$, rarer pyroxenites up to $6 \mathrm{~cm}$ and sporadic pyroxene and spinel megacrysts up to $3 \mathrm{~cm}$ across, as summarised in Everard (2001).

The samples for this mineralogical study were collected by breaking up the dense glassy basalt, which provided more protected, fresher samples than those found in weathered 


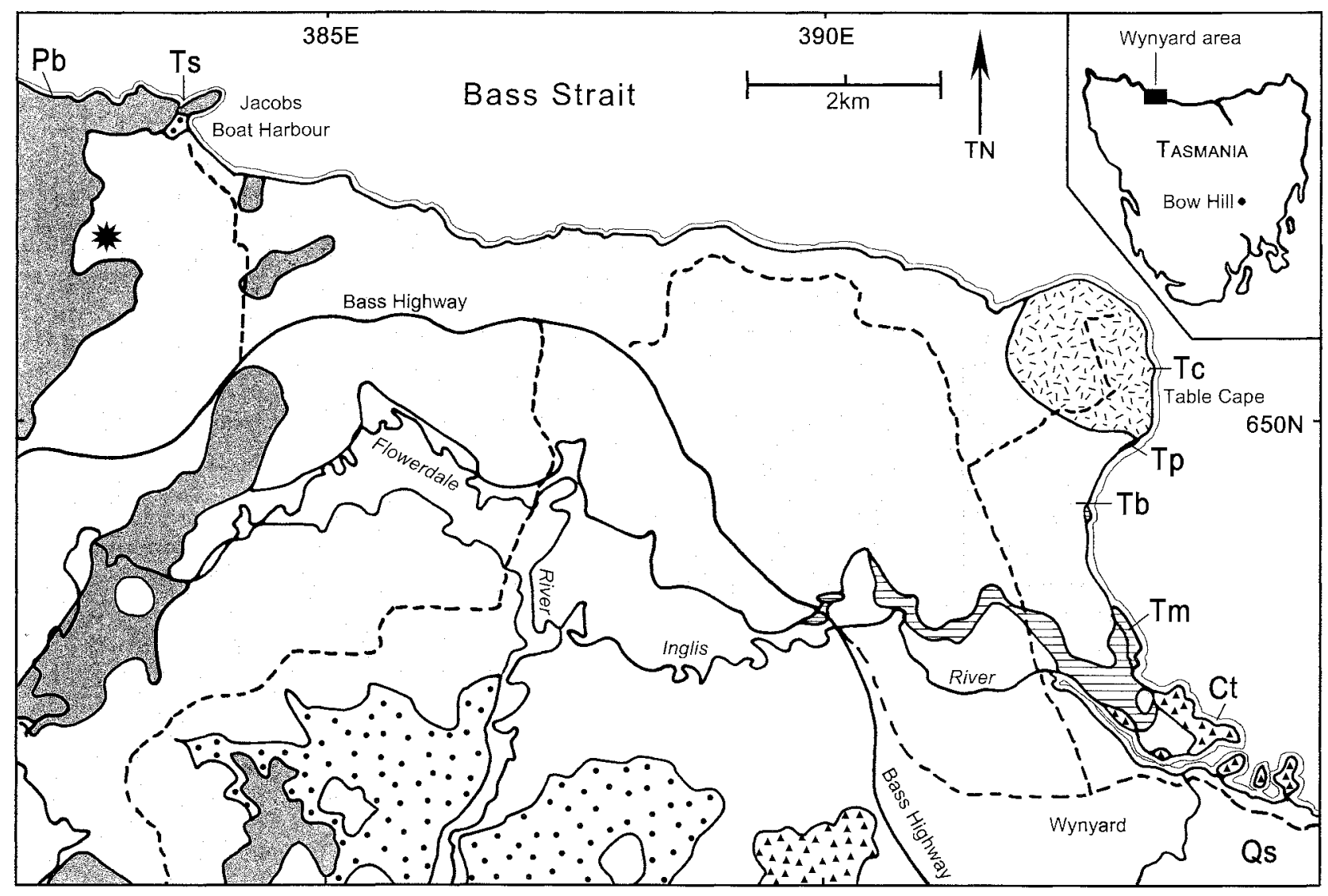

FIG. I - Geological map of the Table Cape-Jacobs Boat Harbour area, west of Wynyard, with Table Cape intrusive core (Tc), Table Cape vent pyroclastics (Tp), Table Cape basalt flows (Tb), underlying Miocene marine beds (Tm) and Carboniferous glacial beds (Ct). Basalt (grey areas) include flows from the Cassidys Creek centre (star) in the western part and overlie Tertiary sands and gravels (Ts) and Precambrian basement beds (Pb). The drainage includes the Flowerdale River (west) which joins the Inglis River with the outlet near Wynyard Creek (east) at Wynyard. The blank land areas include Quaternary alluvium and coastal sands (Qs). Geology after Gee (1971), Sutherland et al. (1996) and Everard (2003).

pyroclastic matrix. Because of the incomplete recovery of xenoliths by this means, a detailed census of xenolith types was not attempted. A range of inclusions was selected for cutting and polishing into discs ready for petrographic microscope examination and analysis by electron microprobe (EMP). The study material is held in the collections of the Australian Museum. The host tachylyte was analysed from hand-picked fragments to avoid included material as much as possible, before crushing for analysis for major, minor and trace elements using conventional X-ray fluorescence (XRF) techniques (table 1 ).

The examination of polished samples by a Leica DMLP polarising microscope with DC 300 digital camera attachment identified a number of xenocryst and xenolith assemblages for detailed analysis and photography (pl. 1). These included:

(a) Pyroxene megacrysts (table 2); clinopyroxene, orthopyroxene.

(b) Metaperidotites (table 3); spinel-metalherzolite, spinelmetaharzburgite.

(c) Metapyroxenites (table 4); metawebsterite, micametawebsterite.

(d) Garnet-metapyroxenites (table 5); garnet ( \pm spinel)metawebsterite.

(e) Garnet-metaclinopyroxenites (table 6); garnet-spinelmetawehrlite, garnet-metaclinopyroxenite. (f) Two-pyroxene granulites (table 7); spinel-granulite, amphibole-mica-granulite.

The assemblages also exhibit secondary breakdown mineral products related to decompression and melt fluid interactions during magmatic transport. These include clinopyroxene, orthopyroxene, spinel and amphibole (table 8) among other phases.

The mineral analyses (tables 2-8) were largely carried out using an ETEC Autoprobe, fitted with three wave-length spectrometers and a LINK EDS system in the Electron Microscope Section, University of Sydney (L. R. Raynor and F. L. Sutherland, analysts). However, some analyses were made on an automated ETEC electron microprobe at Macquarie University, Sydney, for comparative purposes; (B. J. Barron, analyst). Analyses were averaged from multiple spot analyses (up to 10 per grain) for several grains of each mineral phase. The analyses were standardised against natural mineral standards and used $15 \mathrm{kV}$ acceleration voltage, a sample current of $20 \mathrm{nA}$ and Bence-Albee corrections. Precisions were better than $\pm 1 \%$ for element contents above $10 \mathrm{wt} \%$ as oxides, $\pm 5 \%$ at $1-10 \mathrm{wt} \%$ oxides and \pm $10 \%$ at below $1 \mathrm{wt} \%$ oxides. Agreement between analyses for the two instruments was usually within $\pm 2 \%$ for oxides over $5 \mathrm{wt} \%$, within $\pm 8 \%$ for oxides between $1-4 \mathrm{wt} \%$ and within $\pm 30 \%$ for oxides below $1 \mathrm{wt} \%$. 
TABLE 1

Chemical analysis of tachylytic host to Table Cape xenolith suite

\begin{tabular}{|c|c|c|c|c|c|}
\hline \multicolumn{2}{|c|}{ Major elements (wt $\%$ ) } & \multicolumn{2}{|c|}{ Trace elements (ppm) } & \multicolumn{2}{|c|}{ CIPW norm (anhydrous) } \\
\hline $\mathrm{SiO}_{2}$ & 42.46 & $\mathrm{Ni}$ & 278 & $\mathrm{Or}$ & 13.61 \\
\hline $\mathrm{TiO}_{2}$ & 2.32 & $\mathrm{Cr}$ & 360 & $\mathrm{Ab}$ & 3.00 \\
\hline $\mathrm{Al}_{2} \mathrm{O}_{3}$ & 12.39 & $\mathrm{~V}$ & 135 & An & 4.41 \\
\hline $\mathrm{Fe}_{2} \mathrm{O}_{3}$ & 2.15 & $\mathrm{Ba}$ & 181 & $\mathrm{Ne}$ & 22.11 \\
\hline $\mathrm{FeO}$ & 10.74 & $S_{r}$ & 1295 & Di & 25.31 \\
\hline $\mathrm{MnO}$ & 0.20 & $\mathrm{Rb}$ & 14 & $\mathrm{Ol}$ & 20.49 \\
\hline $\mathrm{MgO}$ & 10.13 & Y & 24 & $\mathrm{Mt}$ & 3.18 \\
\hline $\mathrm{CaO}$ & 8.92 & $\mathrm{Ga}$ & 20 & Il & 4.49 \\
\hline $\mathrm{Na}_{2} \mathrm{O}$ & 5.08 & $\mathrm{Zr}$ & 318 & Ap & 3.19 \\
\hline $\mathrm{K}_{2} \mathrm{O}$ & 2.26 & $\mathrm{Nb}$ & 81 & $\mathrm{Cc}$ & 0.28 \\
\hline $\mathrm{P}_{2} \mathrm{O}_{5}$ & 1.32 & $\mathrm{~Pb}$ & 23 & An & 58.1 \\
\hline $\mathrm{CO}_{2}$ & 0.12 & $\mathrm{Zn}$ & 156 & \multirow{4}{*}{\multicolumn{2}{|c|}{$\begin{array}{l}=100 \mathrm{An} / \\
(\mathrm{An}+1.061 \mathrm{Ab}) \\
\text { Differentiation Index } \\
(\mathrm{Or}+\mathrm{Ab}+\mathrm{Ne}) \\
38.7\end{array}$}} \\
\hline $\mathrm{H}_{2} \mathrm{O}+$ & 1.59 & $\mathrm{Cu}$ & 61 & & \\
\hline Total & 99.68 & Th & 7 & & \\
\hline $\mathrm{Mg} /$ & & $\mathrm{U}$ & 4 & & \\
\hline$\left(\mathrm{Mg}+\mathrm{Fe}^{2+}\right) \%$ & 62.70 & & & & \\
\hline
\end{tabular}

Analyst F.L. Sutherland, Macquarie University, Laboratories, Sydney.

(Major elements from $\mathrm{X}$-ray fluorescence spectrometry, using fused borate buttons, with $\mathrm{Na}_{2} \mathrm{O}$ determined by flame photometry, $\mathrm{FeO}$ by dissolution with $\mathrm{HF}$ and ammonium metavanadate and titration against ceric sulphate, with $\mathrm{CO}_{2}$ and $\mathrm{H}_{2} \mathrm{O}$ determined separately: Trace elements from X-ray fluorescence using pressed powder pellets using boric acid bases).

CIPW norm calculated at a ratio of $\mathrm{Fe}_{2} \mathrm{O}_{3} /\left(\mathrm{FeO}+\mathrm{Fe}_{2} \mathrm{O}_{3}\right)=0.2$

\section{RESULTS}

\section{Host Petrology}

The host tachylyte consists of a dark, glassy matrix containing small euhedral, partly corroded olivine (ol) crystals $(-7 \%)$ up to $0.1 \mathrm{~mm}$ across (pl. 1A). These phenocrysts lack the anhedral shapes, strain features and reaction margins observed in the incorporated xenocrysts and xenoliths. Analysis of the tachylyte (table 1) corresponds to a mildly fractionated olivine nephelinite (Mg value $<63$; Ni 278, Cr 360 ppm; Ab $<3 \%$, An mol $\%$ 58, D.I. 39) compared to more primitive Tasmanian nephelinites (Sutherland etal. 2004). The presence of mantle inclusions, however, indicates a mantle-fractionated melt. Normative ol for the Table Cape nephelinite suggests a fractionated composition for the microphenocrystic olivine, which co-crystallised with minute octahedral inclusions of the ulvospinel-magnetite series.

\section{Megacryst Suite}

Clinopyroxene (cpx) is the main megacryst within the Table Cape vent (table 2). Unzoned cores are Al-rich augite $\left(\mathrm{En}_{52-53} \mathrm{WO}_{35-36} \mathrm{Fs}_{11-12}\right)$. They show sieved, resorbed reaction rims composed of $\mathrm{Ti}$ and $\mathrm{Na}$-enriched augite $\left(\mathrm{En}_{44-45} \mathrm{Wo}_{40}\right.$ $\left.\mathrm{Fs}_{15-16}\right)$, which gave low total oxides in EMP analyses due to the inhomogeneous, fluid-bearing melt reactions. A full end-member calculation (Cawthorn \& Collerson 1974) shows the cores are notably more $\mathrm{Mg}$-rich $\left(-\mathrm{En}_{45} \mathrm{Wo}_{24-43}\right.$ $\left.\mathrm{Fs}_{\mathrm{y}_{-10}} \mathrm{Tsc}_{12-13} \mathrm{Jad}_{8-9}, \mathrm{Cta}_{1-2} \mathrm{Cfs}_{1}\right)$ than in most Tasmanian cpx megacrysts (Sutherland et al. 2004). The distinctive megacryst chemistry compared to that of clinopyroxenes in the accompanying xenoliths suggests the former represent a high-pressure liquidus phase crystallised from an $\mathrm{Mg}$-rich parental melt. Experimental work on highly undersaturated nephelinitic or basaltic melts suggests such clinopyroxenes can crystallise as liquidus phases from volatile-bearing melts between $1250-1300^{\circ} \mathrm{C}$ and 2.4-2.6 GPa (Adam 1990).

Orthopyroxene (opx) megacrysts with anhedral, embayed rims and high $\mathrm{Mg}\left(-\mathrm{En}_{88} \mathrm{Fs}_{11} \mathrm{Wo}_{1}\right)$ also occur at Table Cape (table 2). Their compositions, however, closely match those of enstatites in the spinel-metaperidotite xenoliths. Thus, they may derive from these sources rather than from liquidus crystallisation, particularly as opx is commonly reacted out among high pressure liquidus products (Adam 1990). Similarly, the spinel (spl) megacrysts are close to spinel compositions within the xenoliths.

\section{Metaperidotites}

Two distinct types were identified. The more common ( $\mathrm{pl}$. $1 \mathrm{~B})$ is a coarse polygonal-textured assemblage $(-75 \% \mathrm{ol}, 15 \%$ opx, 5\% cpx and 5\% spl from visual grid estimates) with strain/extinction in the silicates. The ol $\left(\mathrm{Fo}_{89}\right)$ and opx $\left(\mathrm{En}_{88}\right)$ are $\mathrm{Mg}$-rich, the cpx is $\mathrm{Al}-\mathrm{Na}$-rich diopside $\left(\mathrm{WO}_{48} \mathrm{En}_{46} \mathrm{Fs}_{6}\right)$ and the spl is $\mathrm{Cr}$-bearing pleonaste (e.g., $\mathrm{Sp}_{78} \mathrm{Hc}_{12} \mathrm{Cm}_{8} \mathrm{Mt}_{1}$, 

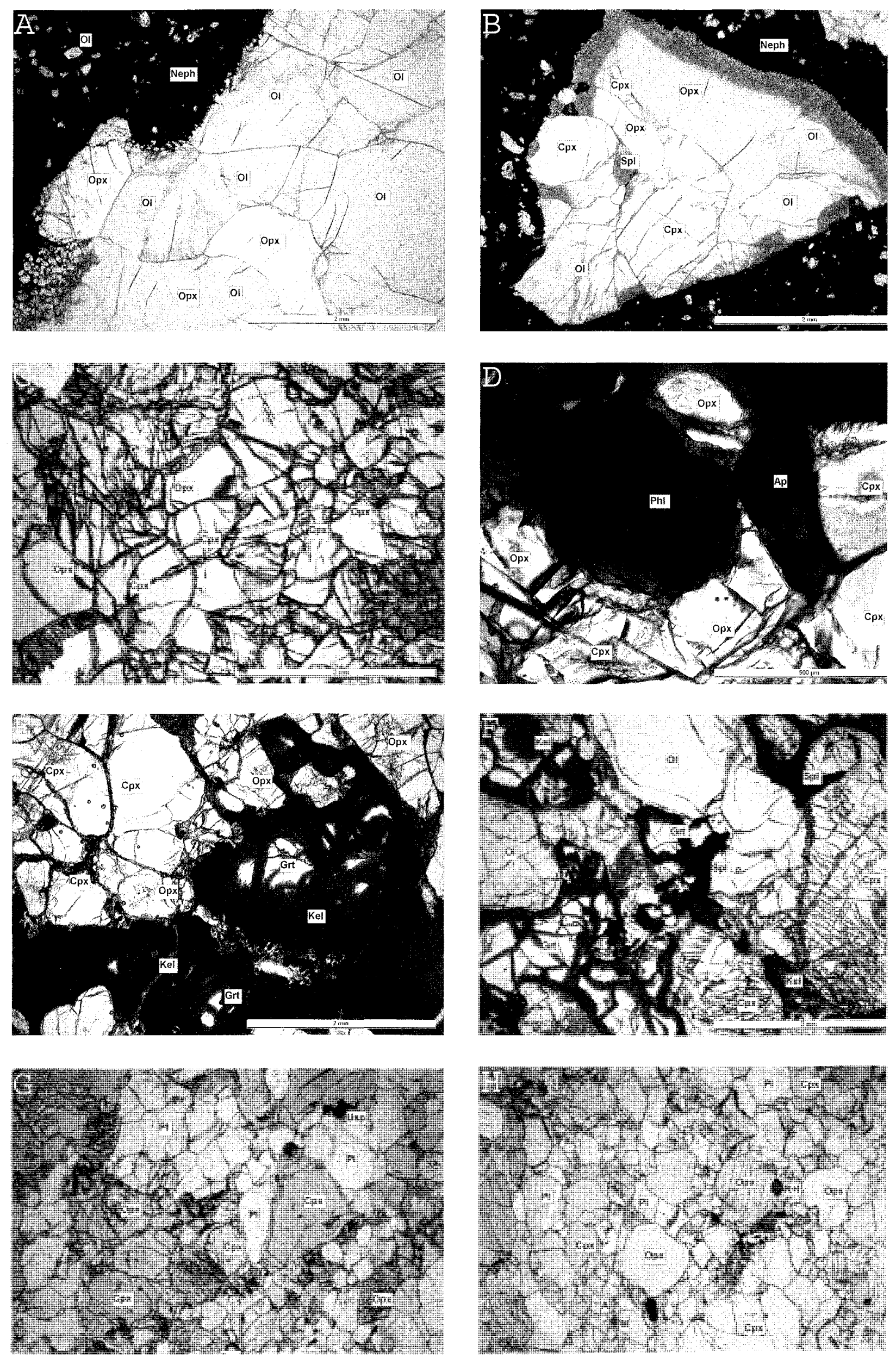
$\mathrm{Usp}_{1}$ ) (table 3). This type dominates mantle xenoliths in the Table Cape region and elsewhere in Tasmania (Piestrzeniewicz 1972, Varne 1977, Sutherland et al. 1996).

Rare spinel-metaharzburgite (pl. 1A) exhibits a medium to coarse granular polygonal texture (grains to $5 \mathrm{~mm}$ across). It is richer in ol (78.5\%) and opx $(20 \%)$ and impoverished in spl (1\%) and $\mathrm{cpx}(0.5 \%)$ compared to the spinel-metalherzolites. The ol $\left(\mathrm{Fo}_{91}\right)$, opx $\left(\mathrm{En}_{89}\right), \mathrm{cpx}\left(\mathrm{En}_{52}\right.$ $\mathrm{WO}_{43} \mathrm{Fs}_{5}$ ) and spl are all $\mathrm{Mg}$-rich phases, with the spl being a Cr-rich magnesiochromite $\left(\mathrm{Mc}_{47} \mathrm{Sp}_{25} \mathrm{Hc}_{22} \mathrm{Mf}_{5} \mathrm{Usp}_{\mathrm{s}}\right)$ (table 3). Such highly depleted metaperidotites are rarely described from Tasmanian mantle xenolith suites.

\section{Metawebsterites}

Two types of metapyroxenites were found. One lacked obvious accessory phases and consisted of subequal polygonal grains of cpx and opx up to $4 \mathrm{~mm}$ across (pl. 1C). Pleochroic cpx ( $\alpha$ pale green, $\beta$ pale brown, $\gamma$ pale pink) is $\mathrm{Al}$-rich, $\mathrm{Na}$-Ti-bearing augite $\left(\mathrm{En}_{48} \mathrm{WO}_{40-41} \mathrm{Fs}_{11-12}\right.$ ) and pleochroic opx ( $\alpha$ pale grey, $\beta$ pale yellow, $\gamma$ pale green) is moderately $\mathrm{Al}$ - and Fe-enriched $\left(\mathrm{En}_{79-81} \mathrm{Fs}_{17-19} \mathrm{WO}_{2}\right)$ (table 4). Exsolution lamellae and blebs of one pyroxene within the other occur in some grains.

The other type is cpx-rich, with large pleochroic cpx grains up to $15 \mathrm{~mm}$ across. These enclose and intergrow with opx, mica and cloudy apatite grains up to $1 \mathrm{~mm}$ across (pl. 1D). The cpx is Al-Mg-rich, Cr-bearing diopside $\left(\mathrm{Wo}_{47} \mathrm{En}_{47}\right.$ $\left.\mathrm{Fs}_{6}\right)$, the opx is Al-bearing enstatite $\left(\mathrm{En}_{88}\right)$, and the mica is Ti-rich magnesian phlogopite (table 4). Such pyroxenites with Cr-diopside suite characteristics (Wilshire \& Shervais 1975) are rare in Tasmania (Everard 2001) and also within eastern Australia (O’Reilly \& Griffin 2000).

\section{Garnet-metawebsterites}

These consist of polygonal, amoeboid-like and blebby intergrowths of cpx, opx, garnet (grt) and in some cases spl, in grains up to $6 \mathrm{~mm}$ across (pl. $1 \mathrm{E}$ ). Smaller areas of finer grained recrystallised mosaics can occur with in coarse-grained matrix. Assemblages carry up to $25 \%$ grt, commonly in relicts within reaction rims and patches but rarely in completely fresh grains. Fresh cores are unzoned pyrope of restricted end-member range ( $\mathrm{Py}_{63-64} \mathrm{Am}_{22-23} \mathrm{Gr}_{9-10} \mathrm{An}_{3-4} \mathrm{Uvo}_{0-1} \mathrm{Sp}_{0-1}$; after Knowles 1987); the pleochroic cpx and opx range from sub-equal to cpx-dominant amounts with only $5 \% \mathrm{opx}$ and include $\mathrm{Al}-\mathrm{Ti}$-Na-rich augite $\left(\mathrm{En}_{45-47} \mathrm{Wo}_{40-41} \mathrm{Fs}_{13-14}\right)$ and Al-rich opx $\left(\mathrm{En}_{77-78}\right)$ (table 5).
Two of the five studied garnet-metawebsterites contain up to $5 \%$ spinel as blocky interstitial grains or as cores up to $1.5 \mathrm{~mm}$ across within reacted garnet enclosures. The spl is $\mathrm{Cr}$-bearing pleonaste $\left(\mathrm{Sp}_{65-72} \mathrm{Hc}_{24-25} \mathrm{Mt}_{2-6} \mathrm{Cm}_{0-3} \mathrm{Usp}_{0-2}\right.$ ) (table 5). Some grains resemble Fe-hydroxide altered olivine $(5 \%)$ in one assemblage, which has pervasive fluid inclusion trails and alterations along grain boundaries. These garnet ( \pm spinel)-two pyroxene assemblages allow TP estimates for the Table Cape vent suite, but are notably more Fe-enriched $\mathrm{cpx}(\mathrm{FeO}$ total $>5 \mathrm{wt} \%$ ) than in most garnet-websterite suites (Sutherland et al. 1994).

\section{Garnet-metaclinopyroxenite and Garnet-metawehrlite}

A rare garnet-metaclinopyroxenite contains abundant reacted garnet $(35 \%)$. This includes and is intergrown with rounded, embayed and polygonal cpx (55\%) grains up to $4.5 \mathrm{~mm}$ across. The reacted areas enclose many secondary spl grains, rimmed by pod-like growths of secondary opx, while al teration prevails along cpx grain boundaries. The grt $\left(\mathrm{Py}_{62} \mathrm{An}_{23} \mathrm{Gr}_{11} \mathrm{An}_{3} \mathrm{Sp}_{1}\right)$ and $\mathrm{Al}-\mathrm{Na}$ - Ti-rich augite $\left(\mathrm{En}_{45} \mathrm{Wo}_{41} \mathrm{Fs}_{14}\right)$ compositions are similar to those in the garnet-metawebsterites and one cpx is mantled by a gedritic amphibole (table 6).

A garnet-metawehrlite contains grt (42.5\%), cpx (37.5\%), spl $(10 \%)$, and ol $(10 \%)$. Poikilitic areas of garnet (to over $12 \mathrm{~mm}$ across) enclose grains and polygonal clusters of the other minerals (pl. 1F). The garnet is pyrope ( $\mathrm{Py}_{63-64} \mathrm{Am}_{22-23}$ $\mathrm{Gr}_{10-11} \mathrm{An}_{3} \mathrm{Sp}_{1}$ ) in composition. The cpx (up to $4.5 \mathrm{~mm}$ across) is Ti-Na-rich augite $\left(E_{n_{46}} W_{o_{42}} F_{12}\right)$, while blocky to rounded $\mathrm{spl}$ grains (up to $1 \mathrm{~mm}$ across) with narrow secondary opx fringes are pleonaste $\left(\mathrm{Sp}_{69-70} \mathrm{Hc}_{22-23} \mathrm{Mt}_{5-6}\right.$ $\left.\mathrm{Usp}_{2} \mathrm{Cm}_{0-1}\right)$ and ol $\left(\mathrm{Fo}_{81}\right)$ forms subhedral grains up to $2.5 \mathrm{~mm}$ across. Other than olivine, the mineralogy (table 6) closely compares with phases in the garnet (spinel)metapyroxenites.

\section{Two-pyroxene Granulites}

These feldspathic rocks form two types. One is a mediumgrained, banded assemblage with conspicuous zones of polygonal plagioclase $(55 \%)$ interspersed with subequal mosaics of pleochroic opx and cpx, mostly $0.5-2.5 \mathrm{~mm}$ across, and sparse interstitial irregular spl grains up to 0.5 $\mathrm{mm}$ across (pl. $1 \mathrm{G})$. The zoned plagioclase shows lamellar and pericline twinning and is calcic $\left(\mathrm{An}_{58-60}\right)$; the cpx is $\mathrm{Al}$ bearing diopside $\left(\mathrm{WO}_{46-47} \mathrm{En}_{36} \mathrm{Fs}_{17-18}\right)$, the opx is $\mathrm{Fe}$-enriched

PLATE I

Photomicrographs of main Table Cape vent xenoliths. (A) Metaharzburgite, in host olivine nephelinite (top left). (B) Spinelmetalherzolite, in host alivine nephelinite. Note reaction rim on xenolith. (C) Metawebsterite, with mosaic-textured pyroxenes. (D) Mica-apatite-bearing metawebsterite. Note basal phlogopite plate (left), cloudy apatite crystal (right) and fuid inclusion trails in the pyroxenes. (E) Gamet-metawebsterite. Note 'kelyphite' alteration rims around inner cores of garnet. (F) Garnetspinel-metawehrlite. Note large fresh olivine grains, 'kelphytised' rims and veins in garnet grains and interstitial spinel grains. (G) Two-pyroxene granulite. Note polygonal texture in plagioclase and pyroxenes and interstitial accessory ulvospinel. (H) Metasomatised two-pyroxene granulite. Note accessory mica, amphibole and rutile grains mantled with ilmenite. All photomicrographs have equal magnification, except for (D) which is enlarged $4 X$. Symbol for host nephelinite is Neph and for minerals are Ol (olivine), Opx (orthopyroxene), Cpx (clinopyroxene), Spl (spinel), Usp (ulvospinel), Grt (garnet), Kel (kelyphite), Pl (plagioclase), Phl (phlogopite), Ap(apatite), A (amphibole), $M$ (mica), R (rutile), I (ilmenite). Microphotographs were taken in transmitted light, I. T. Graham photographer. 
TABLE 2

Representative analyses of pyroxene megacrysts, Table Cape pyroclastics

\begin{tabular}{|c|c|c|c|}
\hline & \multicolumn{2}{|c|}{ Clinopyroxene } & \multirow[t]{2}{*}{ Orthopyroxene } \\
\hline & core & resorbed rim & \\
\hline $\mathrm{SiO}_{2} \mathrm{wt} \%$ & 50.70 & 48.76 & 54.54 \\
\hline $\mathrm{TiO}_{2}$ & 0.61 & 1.36 & 0.12 \\
\hline $\mathrm{Al}_{2} \mathrm{O}_{3}$ & 8.03 & 8.49 & 4.70 \\
\hline $\mathrm{Cr}_{2} \mathrm{O}_{3}$ & 0.25 & - & 0.27 \\
\hline FeO total & 6.52 & 8.26 & 6.87 \\
\hline $\mathrm{MgO}$ & 16.61 & 13.25 & 32.00 \\
\hline $\mathrm{CaO}$ & 15.54 & 16.46 & 0.64 \\
\hline $\mathrm{Na}_{2} \mathrm{O}$ & 1.18 & 1.76 & - \\
\hline Total & 99.44 & 98.34 & 99.14 \\
\hline Cation & $\mathrm{Mg}_{52.8}$ & $\mathrm{Mg}_{44.7}$ & $\mathrm{Mg}_{88 . \mathrm{I}}$ \\
\hline \multirow[t]{2}{*}{ ratio $\%$} & $\mathrm{Ca}_{35.5}$ & $\mathrm{Ca}_{39.9}$ & $\mathrm{Fe}_{10.6}$ \\
\hline & $\mathrm{Fe}_{11.6}$ & $\mathrm{Fe}_{15.5}$ & $\mathrm{Ca}_{13}$ \\
\hline
\end{tabular}

Analyst F. L. Sutherland

TABLE 3

Comparative representative analyses, peridotitic xenoliths (DR 10491)

\begin{tabular}{|c|c|c|c|c|c|c|c|c|}
\hline \multirow{2}{*}{$\begin{array}{l}\text { Mineral/ } \\
\text { Rock Type }\end{array}$} & \multicolumn{2}{|c|}{ Olivine } & \multicolumn{2}{|c|}{ Orthopyroxene } & \multicolumn{2}{|c|}{ Clinopyroxene } & \multicolumn{2}{|c|}{ Spinel } \\
\hline & SML & $\mathrm{SMH}$ & SML & $\mathrm{SMH}$ & SML & $\mathrm{SMH}$ & SML & $\mathrm{SMH}$ \\
\hline $\mathrm{SiO}_{2} \mathrm{wt} \%$ & 40.38 & 41.05 & 53.90 & 55.46 & 50.99 & 52.71 & 0.09 & 0.09 \\
\hline $\mathrm{TiO}_{2}$ & & & 0.15 & 0.08 & 0.74 & 0.11 & 0.18 & 0.12 \\
\hline $\mathrm{Al}_{2} \mathrm{O}_{3}$ & & 0.09 & 4.62 & 2.65 & 6.94 & 3.04 & 60.33 & 26.80 \\
\hline $\mathrm{Cr}_{2} \mathrm{O}_{3}$ & & 0.03 & 0.29 & 0.77 & 0.67 & 1.16 & 7.69 & 40.25 \\
\hline FeO total & 11.09 & 8.95 & 6.89 & 5.70 & 3.01 & 2.89 & 11.14 & 15.44 \\
\hline $\mathrm{MnO}$ & 0.14 & & 0.13 & 0.14 & 0.08 & 0.09 & 0.15 & 0.37 \\
\hline $\mathrm{MgO}$ & 49.29 & 49.31 & 32.28 & 33.36 & 14.40 & 18.12 & 20.61 & 16.29 \\
\hline $\mathrm{CaO}$ & 0.06 & 0.09 & 0.68 & 1.11 & 20.78 & 21.01 & & \\
\hline $\mathrm{Na}_{2} \mathrm{O}$ & & & 0.11 & 0.08 & 1.95 & 0.37 & & \\
\hline Total & 100.96 & 99.52 & 99.05 & 99.35 & 99.56 & 99.50 & 100.19 & 99.36 \\
\hline Cation & $\mathrm{Mg}_{89}$ & $\mathrm{Mg}_{91}$ & $\mathrm{Mg}_{88}$ & $\mathrm{Mg}_{89}$ & $\mathrm{Mg}_{46}$ & $\mathrm{Mg}_{52}$ & $\mathrm{Mg}_{67}$ & $\mathrm{Cr}_{46}$ \\
\hline ratio $\%$ & $\mathrm{Fe}_{11}$ & $\mathrm{Fe}_{9}$ & $\mathrm{Fe}_{11}$ & $\mathrm{Fe}_{9}$ & $\mathrm{Ca}_{48}$ & $\mathrm{Ca} 43$ & $\mathrm{Fe} 20$ & Mg35 \\
\hline & & & $\mathrm{Ca}_{1}$ & $\mathrm{Ca}_{2}$ & $\mathrm{Fe}_{6}$ & $\mathrm{Fe}_{5}$ & $\mathrm{Cr}_{13}$ & $\mathrm{Fe}_{19}$ \\
\hline
\end{tabular}

SML Spinel-metalherzolite SMH Spinel-metaharzburgite. Analyst L. R. Raynor.

Calculated bulk rock compositions, based on modal proportions of mineral phases

\begin{tabular}{lccl} 
Rock & SML & SMH & SML (ol 75\%, opx 15\%, cpx 5\%, spl 5\%) \\
$\mathrm{SiO}_{2}$ & 40.93 & 43.57 & SMH (ol 78.5\%, opx 20\%, spl 1\%, cpx 0.5\%) \\
$\mathrm{TiO}_{2}$ & 0.16 & 0.02 & \\
$\mathrm{Al}_{2} \mathrm{O}_{3}$ & 4.06 & 0.89 & \\
$\mathrm{Cr}_{2} \mathrm{O}_{3}$ & 0.41 & 0.58 & \\
$\mathrm{FeO}$ total & 10.07 & 8.46 & \\
$\mathrm{MnO}$ & 0.14 & 0.03 & \\
$\mathrm{MgO}$ & 43.56 & 45.65 & \\
$\mathrm{CaO}$ & 1.14 & 0.40 & \\
$\mathrm{Na}_{2} \mathrm{O}$ & 0.12 & 0.02 & \\
Total & 100.59 & 99.62 & \\
\hline
\end{tabular}


TABLE 4

Comparative representative analyses, metawebsterites and mica-metawebsterite

\begin{tabular}{|c|c|c|c|c|c|c|c|}
\hline \multirow{2}{*}{$\begin{array}{l}\text { Minerall } \\
\text { rock type }\end{array}$} & \multicolumn{3}{|c|}{ Clinopyroxene } & \multicolumn{3}{|c|}{ Orthopyroxene } & \multirow{2}{*}{$\frac{\text { Mica }}{M W(W W G)}$} \\
\hline & MW(WW I 4) & MW(WW16) & MW(WW6) & MW(WW 14) & MW(WW16) & MW(WW6) & \\
\hline $\mathrm{SiO}_{2} \mathrm{wt} \%$ & 49.85 & 49.87 & 52.08 & 52.71 & 52.83 & 55.01 & 36.69 \\
\hline $\mathrm{TiO}_{2}$ & 1.17 & 1.08 & 0.23 & 0.35 & 0.34 & & 4.38 \\
\hline $\mathrm{Al}_{2} \mathrm{O}_{3}$ & 7.86 & 7.50 & 7.12 & 5.40 & 5.53 & 4.90 & 16.85 \\
\hline $\mathrm{Cr}_{2} \mathrm{O}_{3}$ & 0.29 & 0.40 & 0.75 & 0.17 & 0.23 & 0.24 & 0.49 \\
\hline FeO total & 7.27 & 6.22 & 3.10 & 12.52 & 10.82 & 6.90 & 4.77 \\
\hline $\mathrm{MnO}$ & 0.16 & 0.15 & & 0.19 & 0.18 & & \\
\hline $\mathrm{MgO}$ & 14.21 & 14.41 & 14.28 & 27.70 & 28.55 & 32.11 & 19.87 \\
\hline $\mathrm{CaO}$ & 16.94 & 17.36 & 19.60 & 1.11 & 1.21 & 0.71 & 0.15 \\
\hline $\mathrm{Na}_{2} \mathrm{O}$ & 2.27 & 2.03 & 1.87 & 0.25 & 0.16 & & 0.35 \\
\hline $\mathrm{K}_{2} \mathrm{O}$ & & & & & & & 8.50 \\
\hline Total & 100.02 & 99.02 & 99.03 & 100.40 & 99.85 & 99.87 & 92.05 \\
\hline Cation & $\mathrm{Mg}_{48}$ & $\mathrm{Mg}_{48}$ & $\mathrm{Mg}_{47}$ & $\mathrm{Mg}_{79}$ & $\mathrm{Mgg}_{81}$ & $\mathrm{Mg}_{88}$ & $\mathrm{Mg}_{80}$ \\
\hline \multirow[t]{2}{*}{ ratio $\%$} & $\mathrm{Ca}_{40}$ & $\mathrm{Ca}_{41}$ & $\mathrm{Ca}_{47}$ & $\mathrm{Fe}_{19}$ & $\mathrm{Fe}_{17}$ & $\mathrm{Fe}_{11}$ & $\mathrm{Fe}_{11}$ \\
\hline & $\mathrm{Fe}_{12}$ & $\mathrm{Fe}_{11}$ & $\mathrm{Fe}_{6}$ & $\mathrm{Ca}_{2}$ & $\mathrm{Ca}_{2}$ & $\mathrm{Ca}_{1}$ & $\mathrm{Ti}_{4}$ \\
\hline
\end{tabular}

MW Metawebsterite (WW14, WW16). WW 14 cpx av. 4 analyses, opx av. 4 analyses; WW 16 cpx av. 8 analyses, opx av. 5 analyses. Analyst L. R. Raynor.

MW Mica + apatite-bearing metawebsterite (WW6). Analyst F. L. Sutherland.

Calculated bulk rock compositions, based on modal proportions of mineral phases

\begin{tabular}{|c|c|c|c|c|}
\hline Rock & WW14 & WW16 & WW6 & WW14 (cpx 50\%, opx 50\%) \\
\hline $\mathrm{SiO}_{2} \mathrm{wt} \%$ & 51.28 & 51.36 & 52.33 & $\begin{array}{l}\text { WW } 16(\operatorname{cpx} 50 \%, \text { opx 50\%) } \\
\text { WW6 (cpx 95\%, opx 5\%,phl<0.5\%, }\end{array}$ \\
\hline $\mathrm{TiO}_{2}$ & 0.76 & 0.71 & 0.22 & $a p<0.5 \%)$ \\
\hline $\mathrm{Al}_{2} \mathrm{O}_{3}$ & 6.63 & 6.52 & 7.01 & \\
\hline $\mathrm{Cr}_{2} \mathrm{O}_{3}$ & 0.23 & 0.31 & 0.72 & \\
\hline $\mathrm{FeO}$ total & 9.90 & 8.52 & 3.30 & \\
\hline $\mathrm{MnO}$ & 0.18 & 0.17 & & \\
\hline $\mathrm{MgO}$ & 20.96 & 21.49 & 15.18 & \\
\hline $\mathrm{CaO}$ & 9.03 & 9.29 & 18.66 & \\
\hline $\mathrm{Na}_{2} \mathrm{O}$ & 1.26 & 1.10 & 1.78 & \\
\hline $\mathrm{K}_{2} \mathrm{O}$ & & & $<0.05$ & \\
\hline $\mathrm{P}_{2} \mathrm{O}_{5}$ & & & $<0.05$ & \\
\hline Total & 100.23 & 99.47 & 99.20 & \\
\hline
\end{tabular}


TABLE 5

Comparative representative analyses, garnet-two pyroxene assemblages

\begin{tabular}{|c|c|c|c|c|}
\hline Mineral/rock type & $\begin{array}{c}\text { Clinopyroxene } \\
\text { GW(WW8-Ww13) }\end{array}$ & $\begin{array}{c}\text { Orthopyroxene } \\
\text { GW(WW8-WW13) }\end{array}$ & $\begin{array}{c}\text { Garnet } \\
\text { GW(WW8-WW13) }\end{array}$ & $\begin{array}{c}\text { Spinel } \\
\text { GW(WW12-13) }\end{array}$ \\
\hline $\mathrm{SiO}_{2}$ & $49.47-50.24$ & $51.53-52.41$ & $40.49-41.28$ & \\
\hline $\mathrm{TiO}_{2}$ & $1.28-1.40$ & $0.40-0.50$ & $0.45-0.59$ & $0.76-0.80$ \\
\hline $\mathrm{Al}_{2} \mathrm{O}_{3}$ & $8.05-8.36$ & $5.58-5.98$ & $22.54-22.99$ & $58.26-59.31$ \\
\hline $\mathrm{Cr}_{2} \mathrm{O}_{3}$ & $0.00-0.25$ & $0.03-0.15$ & $0.06-0.40$ & $0.41-2.36$ \\
\hline FeO total & $7.21-7.71$ & $12.46-12.95$ & $12.35-13.66$ & $21.47-22.76$ \\
\hline $\mathrm{MnO}$ & $0.00-0.16$ & $0.15-0.19$ & $0.33-0.41$ & $0.08-0.13$ \\
\hline $\mathrm{MgO}$ & $13.77-14.10$ & $27.26-27.94$ & $16.98-17.45$ & $16.68-16.81$ \\
\hline $\mathrm{CaO}$ & $16.62-16.93$ & $1.12-1.28$ & $5.09-5.26$ & $0.00-0.02$ \\
\hline $\mathrm{Na}_{2} \mathrm{O}$ & $2.05-2.28$ & $0.16-0.24$ & & \\
\hline \multicolumn{5}{|l|}{$\mathrm{K}_{2} \mathrm{O}$} \\
\hline Total & $99.48-100.34$ & $99.68-100.88$ & $99.82-100.53$ & $99.58-100.17$ \\
\hline Cation & $\mathrm{Mg}_{45-47}$ & $\mathrm{Mg}_{77-78}$ & $\mathrm{Mg}_{60-61}$ & $\mathrm{Mg}_{56-60}$ \\
\hline \multirow[t]{2}{*}{ ratio $\%$} & $\mathrm{Ca}_{40-41}$ & $\mathrm{Fe}_{19-20}$ & $\mathrm{Fe}_{26-27}$ & $\mathrm{Fe}_{40-43}$ \\
\hline & $\mathrm{Fe}_{13-14}$ & $\mathrm{Ca}_{2-3}$ & $C a_{13-14}$ & $\mathrm{Cr}_{1-4}$ \\
\hline
\end{tabular}

GW Garnet-websterite ( \pm spinel). WW8-WW10; cpx av. 5-13 analyses, opx av. 4-8 analyses, grt av. 5-6 analyses. GW (WW 12-13) cpx av. 8 analyses, opx av. 4 analyses, grt av. 8 analyses, spinel av. 2-3 analyses. Analyst L. R. Raynor.

$\left(\mathrm{En}_{57-58}\right)$ and the spinel is ulvospinel $\left(\mathrm{Usp}_{92-93} \mathrm{Mf}_{5-6} \mathrm{Sp}_{1-2}\right.$ $\mathrm{Mc}_{0-1}$ ) (table 7).

The other granulite is a fine-grained, slightly foliated assemblage composed of plagioclase (35\%), cpx (30\%) and opx $(25 \%)$ forming strongly polygonal recrystallisations in grains mostly $0.5-1 \mathrm{~mm}$ across ( $\mathrm{pl} .1 \mathrm{H}$ ). The large pyroxenes show strain lamellae and undulose extinction and the cpx is pleochroic. The plagioclase is distinctly calcic $\left(\mathrm{An}_{73-75}\right)$; the cpx is Al-rich diopside $\left(W_{0_{48-49}} E_{n_{40-41}} F_{s_{11-12}}\right)$, the opx is moderately Fe-enriched $\left(\mathrm{En}_{68-70}\right)$ and small interstitial accessory minerals $(10 \%)$ include orange-brown rutile $(5 \%)$ altered on rims and cleavages to opaque $\mathrm{Mg}$-rich ilmenite, pargasitic amphibole, Ti-rich phlogopite and rare corundum (table 7).

\section{Secondary Minerals}

The garnet-bearing assemblages show varied degrees of reaction of the garnet, which alters to cloudy, fine-grained admixtures of spinel and feldspar containing secondary spl, opx and rarely cpx grains. The spl is pleonaste $\left(\mathrm{Sp}_{67-74} \mathrm{Hc}_{23-30}\right.$ $\left.\mathrm{Mr}_{2-3} \mathrm{Usp}_{0-1} \mathrm{Cm}_{0.1}\right)$, the opx is moderately Fe-enriched $\left(\mathrm{En}_{75-}\right.$ $\left.{ }_{79}\right)$ and cpx is Al-rich augite $\left(\mathrm{En}_{49-50} \mathrm{Wo}_{37-38} \mathrm{Fs}_{13}\right)$ (table 8). The Ca-contents in the opx $\left(\mathrm{Wo}_{3-4}\right)$ suggest relatively high temperature reactions were involved in their formation (Brey \& Köhler 1990) while up to $0.7 \% \mathrm{Na}_{2} \mathrm{O}$ in the cpx suggests a metasomatic component helped in the garnet breakdown. The steps involved in such breakdown reactions were detailed in Griffin et al. (1984).

Secondary amphibole forms brown infillings between spl and cpx grains in a garnet-spinel-metaclinopyroxenite. It is kaersutite in composition, distinctly more Ti-rich than the gedritic amphibole that mantles $\mathrm{cpx}$ in the same assemblage, and may derive from fluid infiltrations from the host melt.

\section{Thermobarometry}

Several thermometers and barometers were used to investigate temperature $(\mathrm{T})$ and pressure $(\mathrm{P})$ conditions for metamorphic re-equilibration in the xenolith assemblages. Previously reported couplings (Ellis \& Green $1974 \mathrm{~T}$ and Wood 1974 P; Brey \& Köhler 1990 TP) for Table Cape (Sutherland et al. 1994) are not further considered because of apparent compositional and experimental incompatibilities, discussed later. The Wells (1977) two- pyroxene thermometry, with appropriate allocations of $\mathrm{Fe}^{2+}$ and $\mathrm{Fe}^{3+}$, provides good results at $\mathrm{T}<1300^{\circ} \mathrm{C}$, with uncertainties in the precision within $\pm 50^{\circ} \mathrm{C}$ (Tribaudino \& Bruno 1993, Taylor 1998). This gives a thermal ordering of re-equilibration temperatures (quoted to the nearest $5^{\circ} \mathrm{C}$ ) for the Table Cape vent metaassemblages. The method gives a range of $1055-1070^{\circ} \mathrm{C}$ for garnet ( \pm spinel)-metawebsterites and metawebsterites, $810-1080^{\circ} \mathrm{C}$ for spinel-metaperidotites, $950-960^{\circ} \mathrm{C}$ for mica ( \pm apatite)-metawebsterites and $860-870^{\circ} \mathrm{C}$ for granulites. The relatively wide spinel-metaperidotite temperatures overlap those for the granulites and some metawebsterites and can be refined further using the Sachtleben \& Seck (1981) and Witt-Eickschen \& Seck (1991) thermometric methods. This gave likely $\mathrm{T}$ ranges between $915-1010^{\circ} \mathrm{C}$ for the spinel-lherzolite and $965-1075^{\circ} \mathrm{C}$ for the spinel-bearing harzburgite, which preserves the relative ordering for these assemblages established with the Wells thermometer. The modified metaperidotite $T$ results also delineate the crustal granulite range $\left(<900^{\circ} \mathrm{C}\right)$ from the mantle $\mathrm{T}$ range $\left(>900^{\circ} \mathrm{C}\right)$ and suggest the crust-mantle boundary $(\mathrm{MOHO})$ intersected an ambient geotherm at around $900^{\circ} \mathrm{C}$ (fig. 2).

The garnet-metawebsterites permit three-phase thermobarometry. This study used the Wells $\left(\mathrm{Fe}^{3+}\right)$ two-pyroxene thermometric results with the Wood (1974) garnetorthopyroxene barometer. This coupling provides a narrow $\mathrm{TP}$ range for these assemblages $\left(1050-1070^{\circ} \mathrm{C} ; 1.22-1.36\right.$ GPa), compatible with the tight compositional range for the assemblage analyses and the experimental spinel-pyroxenite/ 
TABLE 6

Comparative representative analyses, garnet-metawehrlite and metaclinopyroxenite

\begin{tabular}{|c|c|c|c|c|c|c|c|c|}
\hline \multirow{2}{*}{$\begin{array}{l}\text { Mineral/ } \\
\text { Rock type }\end{array}$} & \multicolumn{2}{|c|}{ Clinopyroxene } & \multicolumn{2}{|c|}{ Garnet } & \multirow{2}{*}{$\begin{array}{c}\text { Olivine } \\
\text { GW }\end{array}$} & \multicolumn{2}{|c|}{ Spinel } & \multirow{2}{*}{$\frac{\text { Amphibole }}{\mathrm{GC}}$} \\
\hline & GW & $\mathrm{GC}$ & GW & $\mathrm{GC}$ & & GW & $\mathrm{GC}$ & \\
\hline $\mathrm{SiO}_{2}$ & 49.41 & 49.66 & 41.52 & 41.00 & 39.18 & 0.29 & 0.29 & 43.89 \\
\hline $\mathrm{TiO}_{2}$ & 1.47 & 1.43 & 0.43 & 0.49 & & 0.62 & 0.24 & 0.74 \\
\hline $\mathrm{Al}_{2} \mathrm{O}_{3}$ & 9.14 & 8.35 & 23.41 & 23.11 & & 61.01 & 65.17 & 19.01 \\
\hline $\mathrm{Cr}_{2} \mathrm{O}_{3}$ & 0.02 & & 0.03 & & 0.03 & 0.47 & 0.18 & \\
\hline FeO total & 6.65 & 7.68 & 12.08 & 13.61 & 17.49 & 19.62 & 15.66 & 12.67 \\
\hline $\mathrm{MnO}$ & 0.07 & & 0.30 & 0.32 & 0.16 & 0.13 & 0.15 & 0.26 \\
\hline $\mathrm{MgO}$ & 13.66 & 13.84 & 17.70 & 17.08 & 42.96 & 18.20 & 19.02 & 16.63 \\
\hline $\mathrm{CaO}$ & 17.60 & 17.21 & 5.30 & 5.31 & 0.20 & & & 4.00 \\
\hline $\mathrm{Na}_{2} \mathrm{O}$ & 2.07 & 1.98 & & & & & & 0.57 \\
\hline $\mathrm{K}_{2} \mathrm{O}$ & & & & & & & & 0.58 \\
\hline Total & 100.09 & 100.15 & 100.77 & 100.92 & 100.02 & 100.34 & 100.71 & 98.35 \\
\hline Cation & $\mathrm{Mg}_{46}$ & $\mathrm{Mg}_{45}$ & $\mathrm{Mg}_{63}$ & $\mathrm{Mg}_{60 j}$ & $\mathrm{Mg}_{81}$ & $\mathrm{Mg}_{62}$ & $\mathrm{Mg}_{68}$ & $\mathrm{Mg}_{62}$ \\
\hline \multirow[t]{2}{*}{ ratio $\%$} & $\mathrm{Ca}_{12}$ & $\mathrm{Ca}_{41}$ & $\mathrm{Fe}_{24}$ & $\mathrm{Fe}_{27}$ & $\mathrm{Fe}_{19}$ & $\mathrm{Fe}_{37}$ & $\mathrm{Fe}_{32}$ & $\mathrm{Fe}_{27}$ \\
\hline & $\mathrm{Fe}_{12}$ & $\mathrm{Fe}_{14}$ & $\mathrm{Ca}_{13}$ & $\mathrm{Ca}_{13}$ & & $\Gamma \mathrm{i}_{1}$ & $\mathrm{Ca}_{11}$ & \\
\hline
\end{tabular}

GW Garnet-spinel metawehrlite (WW4). Analyst L. R. Raynor. GC Garnet-amphibole-metaclinopyroxenite (WW15).

Analyst B. J. Barron.

Calculated bulk rock compositions, based on modal proportions of mineral phases

\begin{tabular}{lrrl} 
Rock & WW4 & WW15 & WW4 (grt 42.5\%, cpx 37.5\%, spl 10\%, ol 10\%) \\
$\mathrm{SiO}_{2} \mathrm{wt}^{2}$ & 40.13 & 41.66 & WW4 (cpx 55\%, grt 35\%, spl 10\%) \\
$\mathrm{TiO}_{2}$ & 0.79 & 1.00 & \\
$\mathrm{Al}_{2} \mathrm{O}_{3}$ & 19.48 & 19.18 & \\
$\mathrm{Cr}_{2} \mathrm{O}_{3}$ & 0.07 & 0.01 & \\
$\mathrm{FeO}$ total & 11.33 & 10.58 & \\
$\mathrm{MnO}$ & 0.19 & 0.11 & \\
$\mathrm{MgO}$ & 18.76 & 15.59 & \\
$\mathrm{CaO}$ & 8.87 & 11.33 & \\
$\mathrm{Na}_{2} \mathrm{O}$ & 0.78 & 1.09 & \\
$\mathrm{Total}$ & 100.40 & 100.55 & \\
\hline
\end{tabular}




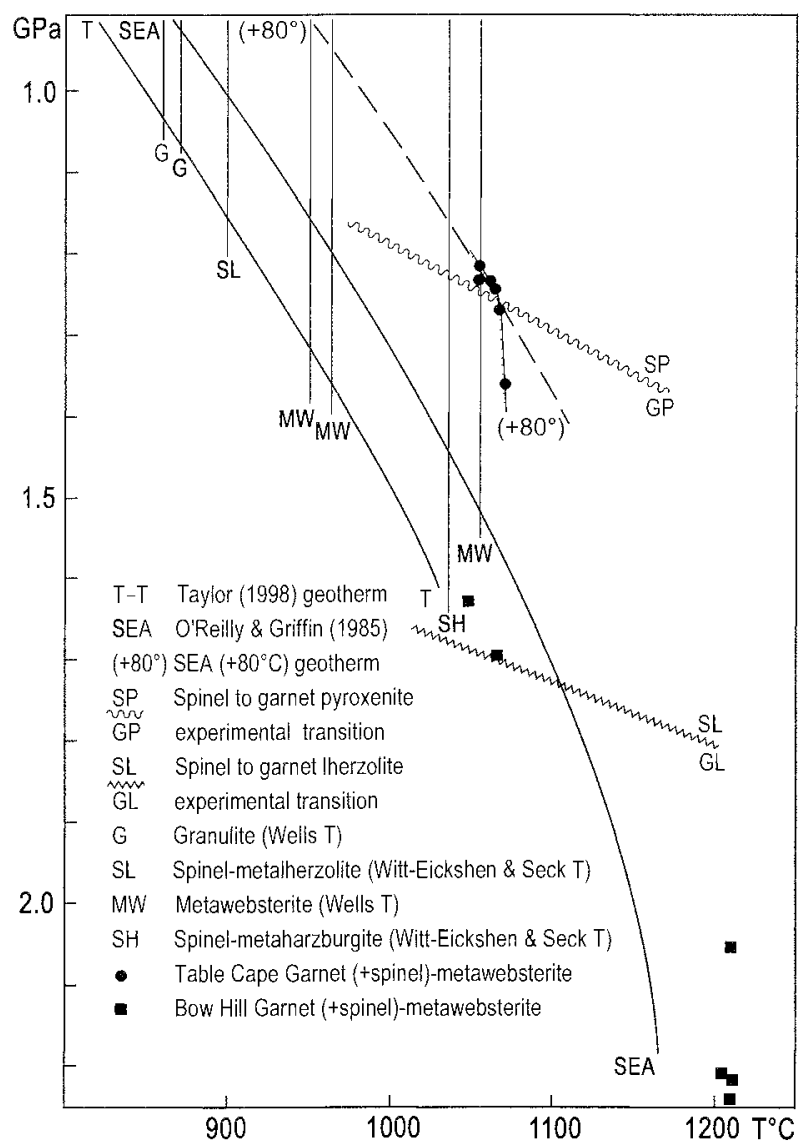

FIG. 2 - Temperature $\left(T^{\circ} \mathrm{C}\right)$-Pressure $(G P a)$ diagram, relating Table Cape (Wells T, Wood P) and Bow Hill (Taylor TP) plots to experimental spinel to garnet pyroxenite and spinel to garnet lherzolite phase transitions. Re-equilibration temperature lines for two-pyroxene granulites and metawebsterites (Wells T) and metaperidotites (Witt-Eickschen \&.Seck T) are indicated to show intersections on different south-eastern Australian geotherms. The TP points for Table Cape garnet ( \pm spinel)-websterites are $1060^{\circ} \mathrm{C}, 1.23 \mathrm{GPa}\left(\mathrm{WW}^{\circ}\right) ; 1065^{\circ} \mathrm{C}, 1.24 \mathrm{GPa}$ (WW9); $1055^{\circ} \mathrm{C}, 1.23 \mathrm{GPa}(\mathrm{WW10}) ; 1070^{\circ} \mathrm{C}, 1.36 \mathrm{GPa}$ (WW12), $1065^{\circ} \mathrm{C}, 1.27 \mathrm{GPa}$ (WW13). The Wood barometry uses the $\mathrm{Cr}-\mathrm{Na}$-Al modification. The TP points for Bow Hill garnet ( \pm spinel)-lherzolites and gamet-websterite are $1048^{\circ} \mathrm{C}, 1.63$ GPa (DRI2508), $1065^{\circ} \mathrm{C}, 1.69 \mathrm{GPa}$ (DR 12501), 1205\% C, $2.21 \mathrm{GPa}(\mathrm{EBH}-\mathrm{E}), 1213^{\circ} \mathrm{C}, 2.22 \mathrm{GPa}(\mathrm{EBH}-\mathrm{D}) ; 1210^{\circ} \mathrm{C}$, 2.24GPa (BHS); $1212^{\circ} \mathrm{C}, 2.05 \mathrm{GPa}$ (QQ20a).

garnet-pyroxenite transition (fig. 2 ). The Table Cape TP results are $60-85^{\circ} \mathrm{C}$ higher at equivalent pressures than for the Southeast Australian (SEA) geotherm of O'Reilly \& Griffin (1985), based on Ellis \& Green (1979)T with Wood (1974) $\mathrm{P}$, and $110-135^{\circ} \mathrm{C}$ higher than for the revised SEA geotherm, using Taylor (1998) TP (fig. 2). This reinforces conclusions from previous thermobarometry for the Table Cape vent suite that suggest a relatively hotter Cenozoic geotherm for the Tasmanian lithosphere (Sutherland et al. 1994), but the new thermobarometry provides a more controlled result.

\section{DISCUSSION}

The Table Cape vent xenoliths allow partial insights into the underlying lower crust-upper mantle lithology and its thermal state when sampled by a Mid-Miocene nephelinitic melt. The host nephelinite was fractionated prior to the eruptive sampling and this evolution took place at TP conditions exceeding those estimated for the deepest contained mantle xenoliths (i.e., $>1070^{\circ} \mathrm{C}$ and $1.4 \mathrm{GPa}$ ).

\section{Host Evolution}

The host nephelinite has generally low $\mathrm{Zr} / \mathrm{Nb}(4.0), \mathrm{Rb} / \mathrm{Sr}$ $(0.017), \mathrm{Ba} / \mathrm{Nb}(2.2)$ and $\mathrm{Ba} / \mathrm{Th}(26)$ ratios, which link it to nephelinites of the low Rb and Ba group in Tasmania (e.g., West Scottsdale, Northeast Tasmania; see Sutherland et al. 2004). These particular nephelinites are attributed to low degrees of partial manrle melting in which some phlogopite is retained in the mantle source. In this respect, the Table Cape vent nephelinite differs from the nearby, but older, nephelinite at Boat Harbour, which relates to the high Ba and Rb group (Sutherland et al. 1996). Similar nephelinites to the Table Cape vent melt represent initial melts with liquidus temperatures around $1250-1300^{\circ} \mathrm{C}$ at $2.4-3.5 \mathrm{GPa}$ based on experimental work on Tasmanian examples from Lughrata and Scottsdale (Tilley \& Thompson 1972, Frey et al. 1978, Adam 1990). The experiments on the Scottsdale nephelinite suggest such melts with only slightly (1-2 wt \%) higher $\mathrm{SiO}_{2}$ can be generated from garnet lherzolite mantle that contains volatile $\left(\mathrm{H}_{2} \mathrm{O}+\mathrm{CO}_{2}\right)$ concentrations of 6.5 wt $\%$ at $3.4 \mathrm{GPa}$ (Adam 1990).

The precise high pressure mineral phases that crystallised during evolution of the erupted Table Cape vent melt are difficult to completely designate. They probably include clinopyroxene similar to the $\mathrm{Mg}$-rich augite megacrysts (see Adam 1990, fig. 7), Other potential phases such as olivine, spinel and garnet may be difficult to distinguish in composition from those released from disaggregated mantle xenoliths, although some cases may be distinguished texturally and based on inclusions.

\section{Assemblage Affinities}

The xenoliths include mantle metaperidotites and metapyroxenites and crustal granulites, but their chemical characterisations are handicapped by lack of sufficiently large and fresh samples for analysis within the altered pyroclastic deposits. Only garnet-metapyroxenite and granulites were found suitable for conventional analyses (Sutherland et al. 2004). Approximate bulk compositions, however, were calculated from small, fresh samples by using estimated proportions of mineral phases analysed by EMP, using a visual grid.

The metaperidotites show significant olivine alteration in larger xenoliths, but compositions were estimated from mineral analyses in fresh spinel-metalherzolite and metaharzburgite (table 3). These suggest the metalherzolite is enriched in $\mathrm{Al}, \mathrm{Fe}, \mathrm{Ca}$ and $\mathrm{Na}$ and depleted in $\mathrm{Si}$ relative to the harzburgite. 'Mantle' CIPW norms based on the analyses, after Wilkinson \& Stolz (1997), gave reasonable matches with the modal mineralogy, showing higher Ca-tschermakite in the metalherzolite (Cats 4.4 of $1.5 \mathrm{wt} \%$ ) and higher $\mathrm{Hy}$ in the metaharzburgite (22.8 of $13.5 \mathrm{wt} \%)$. The higher 
TABLE 7

Comparative representative analyses, two-pyroxene granulites

\begin{tabular}{|c|c|c|c|c|c|c|c|c|}
\hline \multirow{2}{*}{$\begin{array}{l}\text { Mineral/ } \\
\text { rock type }\end{array}$} & \multicolumn{2}{|c|}{ Clinopyroxene } & \multicolumn{2}{|c|}{ Orthopyroxene } & \multicolumn{2}{|c|}{ Plagioclase } & \multirow{2}{*}{$\begin{array}{l}\text { Amphibole } \\
\text { MG(WW3) }\end{array}$} & \multirow{2}{*}{$\frac{\text { Mica }}{M G(W W 3)}$} \\
\hline & $\mathrm{BG}(\mathrm{WW} 1)$ & MG(WW3) & BG(WW1) & MG(WW3) & $B G(W W 1)$ & $M G(W W 3)$ & & \\
\hline $\mathrm{SiO}_{2} \%$ & 50.99 & 50.71 & 50.80 & 52.67 & 53.58 & 49.69 & 42.34 & 37.52 \\
\hline $\mathrm{TiO}_{2}$ & 0.22 & 0.56 & 0.14 & & & & 2.92 & 5.45 \\
\hline $\mathrm{Al}_{2} \mathrm{O}_{3}$ & 3.70 & 4.94 & 1.79 & 2.49 & 29.78 & 32.84 & 14.27 & 15.82 \\
\hline $\mathrm{Cr}_{2} \mathrm{O}_{3}$ & & 0.23 & & & & & 0.27 & \\
\hline $\mathrm{FeO}$ total & 10.53 & 6.57 & 26.25 & 19.49 & & & 10.32 & 10.52 \\
\hline $\mathrm{MnO}$ & 0.15 & & 0.51 & 0.23 & & & & \\
\hline $\mathrm{MgO}$ & 12.15 & 13.35 & 20.09 & 24.24 & & & 13.35 & 14.78 \\
\hline $\mathrm{CaO}$ & 21.79 & 22.12 & 0.43 & 0.54 & 12.08 & 15.11 & 11.69 & 3.23 \\
\hline $\mathrm{Na}_{2} \mathrm{O}$ & 0.47 & 0.48 & & & 4.64 & 2.86 & 1.57 & 0.65 \\
\hline $\mathrm{K}_{2} \mathrm{O}$ & & & & & 0.11 & 0.21 & 2.11 & 6.00 \\
\hline Total & 100.00 & 98.96 & 100.01 & 99.66 & 100.19 & 100.71 & 98.84 & 93.97 \\
\hline Cation & $\mathrm{Ca}_{46-17}$ & $\mathrm{Ca}_{48-49}$ & $\mathrm{Mg}_{57-58}$ & $\mathrm{Mg}_{68-69}$ & $\mathrm{Ca}_{58-59}$ & $\mathrm{Ca}_{7374}$ & $\mathrm{Mg}_{48-49}$ & $\mathrm{Mg}_{6,3}$ \\
\hline racio $\%$ & $\mathrm{Mg}_{36}$ & $\mathrm{Mg}_{40-41}$ & $\mathrm{Fe}_{41-\{2}$ & $\mathrm{Fe}_{30-31}$ & $\mathrm{Na}_{40-41}$ & $\mathrm{Na}_{25-26}$ & $\mathrm{Ca}_{30-31}$ & $\mathrm{Fe}_{25}$ \\
\hline & $\mathrm{Fe}_{17-18}$ & $\mathrm{Fe}_{11-12}$ & $\mathrm{Ca}_{1}$ & $\mathrm{Ca}_{1}$ & $\mathrm{~K}_{0-1}$ & $\mathrm{~K}_{1-2}$ & $\mathrm{Fe}_{21}$ & $T_{12}$ \\
\hline
\end{tabular}

BG Banded granulite (WW1), includes ulvospinel $\left(\mathrm{Fe}_{66} \mathrm{Ti}_{29} \mathrm{Mg}_{5}\right.$ ). $\mathrm{MG}$ Micro-granulite (WW3), includes rutile with Mg-rich ilmenite rims $\left(\mathrm{Ti}_{5_{50}} \mathrm{Fe}_{20} \mathrm{Mg}_{21}\right)$. Analyst F. L. Sutherland.

TABLE 8

Comparative representative analyses, secondary phases, garnet-pyroxene and pyroxene assemblages

\begin{tabular}{|c|c|c|c|c|c|c|c|c|c|}
\hline $\begin{array}{l}\text { Mineral/ } \\
\text { rock type }\end{array}$ & $\begin{array}{c}\text { Cpx } \\
\text { WW4 }\end{array}$ & $\begin{array}{c}\text { Opx } \\
\text { WN4 }\end{array}$ & $\begin{array}{c}\text { Opx } \\
\text { WW9 }\end{array}$ & $\begin{array}{c}\text { Opx } \\
\text { WW15 } \\
\end{array}$ & $\begin{array}{c}\text { Spl } \\
\text { WW9 }\end{array}$ & $\begin{array}{c}\text { Spl } \\
\text { WW12 }\end{array}$ & $\begin{array}{c}\text { Spl } \\
\text { WW14 }\end{array}$ & $\begin{array}{c}\text { Spl } \\
\text { WW15 } \\
\end{array}$ & $\begin{array}{c}\text { Amp } \\
\text { WW15 }\end{array}$ \\
\hline $\mathrm{SiO}_{2} \mathrm{wt} \%$ & 48.36 & 52.17 & 48.46 & 50.06 & 0.25 & & & 0.34 & 36.12 \\
\hline $\mathrm{TiO}_{2}$ & 0.68 & 0.23 & 0.39 & 0.54 & 0.21 & 0.14 & 0.22 & 0.19 & 5.45 \\
\hline $\mathrm{Al}_{2} \mathrm{O}_{3}$ & 11.00 & 8.17 & 11.00 & 10.81 & 63.98 & 65.08 & 64.53 & 65.00 & 6.84 \\
\hline $\mathrm{Cr}_{2} \mathrm{O}_{3}$ & 0.04 & 0.04 & 0.22 & 0.02 & 1.18 & 0.39 & 0.35 & 0.13 & 0.02 \\
\hline FeO total & 7.17 & 11.03 & 12.27 & 10.50 & 16.48 & 15.02 & 17.27 & 14.85 & 19.70 \\
\hline $\mathrm{MnO}$ & 0.28 & 0.27 & 0.36 & 0.27 & 0.23 & 0.13 & 0.17 & 0.16 & 0.28 \\
\hline $\mathrm{MgO}$ & 15.34 & 26.82 & 24.95 & 26.66 & 18.30 & 19.17 & 17.85 & 19.55 & 13.28 \\
\hline $\mathrm{CaO}$ & 16.11 & 1.86 & 1.68 & 1.75 & & & & & 6.80 \\
\hline $\mathrm{Na}_{2} \mathrm{O}$ & 0.68 & 0.18 & 0.09 & 0.11 & & & & & 1.22 \\
\hline $\mathrm{K}_{2} \mathrm{O}$ & & & & & & & & & 1.50 \\
\hline Total & 99.66 & 100.77 & 99.42 & 100.72 & 100.63 & 99.93 & 100.39 & 100.22 & 91.21 \\
\hline Cation & $\mathrm{Mg}_{49-50}$ & $\mathrm{Mg}_{78}$ & $\mathrm{Mg}_{75,76}$ & $\mathrm{Mg}_{7877}$ & $\mathrm{Mg}_{64-65}$ & $M g_{69-70}$ & $\mathrm{Mg}_{64-65}$ & $\mathrm{Mg}_{70}$ & $\mathrm{Mg}_{4 ;-16}$ \\
\hline \multirow[t]{2}{*}{ ratio \% } & $\mathrm{Ca}_{37-38}$ & $\mathrm{Fe}_{18}$ & $\mathrm{Fe}_{2(1-21}$ & $\mathrm{Fe}_{1>18}$ & $\mathrm{Fe}_{31-32}$ & $\mathrm{Fe}_{30-31}$ & $\mathrm{Fe}_{35}$ & $\mathrm{Fe}_{30}$ & $\mathrm{Fe}_{3-3,3}$ \\
\hline & $\mathrm{Fe}_{13}$ & $\mathrm{Ca}_{4}$ & $\mathrm{Ca}_{3-4}$ & $\mathrm{Ca}_{3-4}$ & $\mathrm{Cr}_{4-5}$ & $\mathrm{Ti}_{0-1}$ & $\mathrm{Cr}_{0-1}$ & $T i_{01}$ & $\mathrm{Ca}_{16,17}$ \\
\hline
\end{tabular}

Cpx (dinopyroxene). Opx (orthopyroxene). Spl (spinel). Amp (amphibole)

WW4 Garnet-metawehrlite. WW9 Garnet-metawebsterite. WW12 Garnet-spinel-metawebsterite. WW14. Metaclinopyroxenite. WW15

Garnet-amphibole-metawebsteritc. Analyst L. R. Raynor. 
$\mathrm{Mg} /\left(\mathrm{Mg}+\mathrm{Fe}^{2+}\right)$ for the metaharzburgite (0.923) compared to metalherzolite $(0.906)$ indicates its more depleted nature. Two potential sources exist for the metaharzburgite xenolith. One is a highly-depleted mantle component, while the other is a higher level fragment derived from an underlying, unexposed Cambrian ultramafic complex as seen outcropping elsewhere in northwestern Tasmania (Brown 1988). Cambrian harzburgites, however, contain $\mathrm{Cr}$-rich spinels with higher $100 \mathrm{xCr} /(\mathrm{Cr}+\mathrm{Al})$ and $100 \mathrm{xMg} /\left(\mathrm{Mg}+\mathrm{Fe}^{2+}\right)$ values (Brown 1988, fig. 8) than the Table Cape vent metaharzburgite ( $\mathrm{Cr}$ value $0.50, \mathrm{Mg}$ value 0.65 ). This favours a separate origin for the metaharzburgite, which although a scarce lithology in Southeast Australian mantle peridotite suites, can be significant at rare sites (e.g., Mt Leura, Victoria; Chen et al. 1989). The calculated bulk analysis and mineralogy for the Table Cape vent metaharzburgite match those of the high $\mathrm{Mg}$ value $(0.92-0.93) \mathrm{Mt}$ Leura harzburgites, rather that the lower $\mathrm{Mg}$ value $(0.87-0.90)$ harzburgites that Chen et al. (1989) interpreted as cumulates. The Table Cape vent metaharzburgite pyroxenes have higher $\mathrm{Ca}$ in the opx and lower $\mathrm{Na}$ and $\mathrm{Al}$ in the cpx than the Mt Leura examples. They seem a deeper, higher $T$ variant than at Mt Leura where they dominate in the uppermost mantle zone.

Garnet-metawebsterites dominate the Table Cape vent garnet-metapyroxenites. A bulk analysis gives a relatively low Si, high Mg composition (Sutherland et al. 2004) but with significantly lower $\mathrm{Mg} /\left(\mathrm{Mg}+\mathrm{Fe}^{2+}\right)$ than for the metaperidotites $(0.79$ of 0.90$)$. The 'mantle' norm is dominated by $\mathrm{Hy}(37 \%)$ and Cats $(33 \%)$, with subordinate Ol $(12 \%)$. This suggests a recrystallised mafic parental cumulate, either from transitional olivine basalt or olivine tholeiite composition. The high $\mathrm{Ni}(419 \mathrm{ppm})$ and $\mathrm{Cr}$ (387 ppm) and low $\mathrm{Zr}$ (47 ppm), Ba (7 ppm) and Rb (3 $\mathrm{ppm})$ are compatible with a relatively mafic cumulate or little fractionated basalt melt. Although high $\mathrm{Ni}$ and $\mathrm{Cr}$ can be introduced by mantle wall rock-melt interactions (O'Reilly et al. 1989), this is considered unlikely here as no composite xenoliths are evident. The present neoblastic assemblage of cpx, opx, spl and grt formed from progressive recrystallisation of an original magmatic parent along reactions similar to those outlined from western Victorian suites (O'Reilly et al. 1989). The rarer wehrlites and olivinebearing clinopyroxenite at Table Cape vent may represent less re-constituted lithologies or possibly assemblages derived from more alkaline parental melts. Their calculated bulk compositions (table 6) and 'mantle' norms (Cats 34-37, Hy 35-37, Ol 9-16 wt\%) overlap those of garnet-metawebsterites. The Table Cape garnet-metapyroxenites are essentially 'dry' assemblages and differ from the hydrous amphibole-micabearing suites in some SE Australian xenolith suites (e.g., Bullenmerri, Gnotuk, Anakie East; Graham et al. 2003). They also differ in mineral and bulk compositions from 'dry' garnet-metapyroxenite suites that typify many eastern Australian suites (Irving 1974, Stolz 1984, Griffin et al. 1987), in being more Fe-rich. Relatively little experimental work is available on Australian garnet-metawebsterites, except for an Fe-poor Delegate example (Irving 1974). This work gave a TP point for the co-existing grt, cpx and opx at $1200^{\circ} \mathrm{C}$ and $1.5 \mathrm{GPa}$, which lies on the high $\mathrm{T}$ side (fig. 2) of the Table Cape vent Fe-rich metawebsterite re-equilibration conditions $\left(1055-1100^{\circ} \mathrm{C}, 1.2-1.4 \mathrm{GPa}\right)$.

Metawebsterites, lacking garnet, form a minor component at Table Cape vent and include two distinct types. The more common type consists of a polygonal-textured pyroxene mosaic with opx compositions that overlap those in the garnet ( \pm spinel)-metawebsterites (see tables 4 and 5 ). This suggests links between these two assemblages. Calculated bulk analyses, however, are notably higher in $\mathrm{Si}$ and $\mathrm{Mg}$ and lower in $\mathrm{Al}$ and $\mathrm{Fe}$ for the metawebsterites (table 4) and give 'mantle' norms with high $\mathrm{Hy}(51 \mathrm{wt} \%)$, moderate Di $(26 \mathrm{wt} \%)$, minor Cats $(10 \mathrm{wt} \%$ ) and negligible or no Ol. This suggests a tholeiitic parental affinity, unless the metawebsterites are only pyroxene-rich parts from inhomogeneous garnet $( \pm$ spinel)-metawebsterite bodies. Alternatively they may be recrystallisations of coarse subcalcic clinopyroxenites, originally crystallised as liquidus precipitates, as proposed for some metawebsterite xenoliths near Glen Innes, New South Wales (Wilkinson \& Stolz 1997). This origin is favoured by mutual unmixing in the pyroxenes and a bulk recalculated composition close to that of a subcalcic clinopyroxene. The Table Cape vent metawebsterites, however, have lower $\mathrm{Ca}$ and higher $\mathrm{Ti}$ than in the Glen Innes subcalcic clinopyroxene (table 4, of with Wilkinson \& Stolz, tables 5 and 10). In contrast, the other type of Table Cape vent metawebsterites approach $\mathrm{Cr}$ bearing diopside in composition (table 4) and are higher in $\mathrm{Ca}$ and lower in $\mathrm{Mg}$ and $\mathrm{Fe}$ than the $\mathrm{Ti}$-Al-rich websterites. The accessory Ti-bearing phlogopite and apatite suggest an introduced metasomatic component enriched in $\mathrm{Ti}, \mathrm{K}$ and $\mathrm{P}$. The cloudy apatite resembles that in diopsidic vein material within metasomatised metalherzolites in southeastern Australia (O'Reilly \& Griffin 2000). Such mantle apatites in southeastern Australian xenoliths come from a relatively young metasomatic event, originally assigned to a deep (plume-related) fluid. Re-examination of the apatite neon isotope data, however, now suggests it derives from Mid-Ocean Ridge Basalt (MORB) fluids rather than a primitive plume source (Gautheron \& Moreira 2003).

The crustal granulites are two-pyroxene, Ca-rich feldspar assemblages and include both anhydrous and hydrous metasomatised types. An anhydrous banded granulite (Sutherland et al. 2004) corresponds chemically to a transitional olivine tholeiite. It equates to a basalt protolith $\left(\mathrm{SiO}_{2} 51\right.$, Di 25, Hy 22, Ol $\left.4 \mathrm{wt} \%\right)$ of relatively unfractionated nature, with $\mathrm{Mg} /\left(\mathrm{Mg}+\mathrm{Fe}^{2+}\right)=0.67$ and $\mathrm{An} /(\mathrm{An}+\mathrm{Ab})=0.59$. The hydrous micro-granulite exhibits a diverse accessory mineralogy that includes Ti-bearing amphibole and mica, rutile-ilmenite and rare corundum. It is even more $\mathrm{Mg}$-rich and primitive in its features than the anhydrous granulite, with $\mathrm{Mg} /\left(\mathrm{Mg}+\mathrm{Fe}^{2+}\right)=0.74$ and $\mathrm{An}_{n} /(\mathrm{An}+\mathrm{Ab})=0.68$, high $\mathrm{Ni}+\mathrm{Cr}(522 \mathrm{ppm})$ and low $\mathrm{Zr}(53$ $\mathrm{ppm})$ contents. It also corresponds to transitional olivine basalt in its $\mathrm{SiO}_{2}(50 \mathrm{wt} \%)$ and orher normative contents (Hy 32, Di 9, Ol 2 wt \%). The accessory minerals and textures suggest modification of a basaltic parent by volatile, $\mathrm{Al}, \mathrm{Ti}, \mathrm{Fe}$ and K-bearing fluids prior to metamorphic reequilibration.

The garnet-metawebsterites and two-pyroxene granulites in the Table Cape vent suite suggest transitional olivine basalt protoliths were emplaced at both mantle and crustal levels. Normative wt $\%$ ratios for these rocks (Hy 44-75, Di 20-48, Ol 8-23) plot across relatively unoccupied positions in the Ol-Di-Hy normative tetrahedron for eastern Australian assemblages (O'Reilly et al. 1989). This supports an unusual lithospheric section below Table Cape, in keeping with the moderately Fe-rich pyroxene mineralogy in these assemblages. The Table Cape vent calcic plagioclase granulites differ from granulite in the Boat Harbour section, which contains Na-rich plagioclase (Sutherland et al. 1996). 
Although more silica-saturated protoliths dominate the Table Cape vent $\mathrm{Al}$-Ti-bearing augite suites, rare metawehrlite and garnet-metaclinopyroxenites suggest more olivine-rich and perhaps even alkaline progenitors may have been present.

\section{Tasmanian/East Australian Geotherms}

TheSEA geotherm was established using Ellis \& Green (1979) grt-cpx exchange thermometry, which, while accommodating $\mathrm{Fe}^{2+}$ and $\mathrm{Fe}^{3+}$ assignments in the minerals, generally leads to wider temperature differences between these allocations than does the Wells thermometry (Griffin et al. 1984, O'Reilly \& Griffin 1985). The Ellis \& Green thermometer, however, has proved unreliable in its application. It generally overestimates $T$ relative to experimental results for the TP range for such xenolith suites (see Sutherland et al. 1994, 1998, Ai 1994 , Taylor 1998) or is erratic where Ca exceeds a concentration of 0.15 atoms in the garnet (Alaoui et al. 1997). The Table Cape vent garnets all have $\mathrm{Ca}<0.15$, but application of Ai (1994) thermometry nevertheless gives highly erratic results. For these reasons, grt-cpx exchange thermometry is abandoned for the Table Cape vent grt-bearing assemblages, in favour of Wells thermometry. The comparison in T range between Ellis \& Green $\left(\mathrm{Fe}^{3+}\right)$ and Wells $\left(\mathrm{Fe}^{3+}\right)$ for Table Cape vent grt-websterites is $1005-1220^{\circ} \mathrm{C}$ and $1055-1075^{\circ} \mathrm{C}$ respectively, which leads to corresponding differences in P estimates when inserted into the Wood barometer (e.g., 0.74-1.86 GPa, Sutherland et al. 1994, of 1.23-1.36 GPa, Sutherland et al. 2004).

Wood (1974) barometry, however, is retained for the Table Cape vent study as a suitable barometer for Fe-rich grt-metawebsterites, particularly as some other potential barometers were experimentally calibrated against more Mg-rich assemblages. This includes Taylor (1998) thermobarometry, which provides a lower intersection than the original SEA gradient. The new Table Cape vent thermobarometry indicates a coherent grouping for grtwebsterites near the base of the sampled mantle section at temperatures well above SEA (fig. 2 ). The Table Cape vent TP section extends to $45 \mathrm{~km}$ depth, so represents a higher level section than at Bow Hill, Tasmania, which extends into the spinel-garnet-metalherzolite transition.

The Mg-rich Bow Hill grt-assemblages (Sutherland et al. 1984) need different thermobarometry than for Table Cape assemblages. With Wells (1977) thermometry (with only $\mathrm{Fe}^{2+}$ allocated) and Wood (1974) barometry, the wide range of results $\left(1000-1180^{\circ} \mathrm{C}, 1.0-2.5 \mathrm{Gpa}\right)$ extend well beyond the experimental garnet-spinel lherzolite transition zone, suggesting an unsuitable coupling. The Taylor (1998) TP method, which gives T estimates closest to Wells T, was chosen as a more appropriate coupling. In calculating TP, $\mathrm{Fe}^{2+}$ and $\mathrm{Fe}^{3+}$ was allocated in the garnets (Ca concentration 0.12 to 0.14 atoms) by charge balance and in the pyroxene by the cation allocation method of Cawthorn \& Collerson (1974). The results (I. Roach, Australian National University, pers. comm. 2004) range from $1048-1065^{\circ} \mathrm{C}, 1.63-1.69$ $\mathrm{GPa}$ (grt-spl-metalherzolite) to $1205-1213^{\circ} \mathrm{C}, 2.21-2.24$ $\mathrm{GPa}$ (grt-metalherzolite) and $1213^{\circ} \mathrm{C}$ and $2.05 \mathrm{GPa}$ (grtmetawebsterite). These results lie on the high $\mathrm{T}$ side of the Taylor (1998) modified SEA geotherm ('T-T, fig. 2) and the higher $\mathrm{P}$ assemblages even lie above the original SEA geotherm. Thus, both Table Cape and Bow Hill suites suggest that extreme geothermal gradients existed in the Tasmanian Miocene lithosphere.
The precise profiles and extent of variations within Mesozoic-Cainozoic East Australian geotherms have engendered debate as to whether the TP profiles overestimate the data or mark more conservative profiles, depending on the choice of thermometer/barometer couplings and other effects (McDonough et al. 1991, Sutherland et al. 1994, 1998, O'Reilly et al. 1997, Sutherland 1999, Gaul et al. 2003, Roach 2004). Various protocols for thermometer and barometer usage in general, that incorporate differences in mineralogical compositions of assemblages, have been proposed (O'Reilly et al. 1997, Xu et al. 1998, Smith 1999). These suggested couplings do not include some well-based newer couplings (Taylor $1998 \mathrm{TP}$ ). Thus, uncertainties exist in geotherm comparisons for the East Australian/East Asian xenolith suites. In a study of xenoliths in Miocene basalts from northern China, Chen et al. (2001) preferred Brey \& Köhler (1990) TP for the Mg-rich garnet-websterites, rather than the Ellis \& Green (1979) and Wood (1974) TP, and the results clearly plot well above the SEA geotherm which was based on the latter coupling. However, they retained Ellis \& Green T and Wood P for an Fe-rich garnet-metawebsterite, which placed this plagioclase-bearing assemblage at over $70 \mathrm{~km}$ depth, well-divorced from the other TP plots.

Despite the complications in selecting thermobarometric methods, the two revised Tasmanian Miocene geotherms both suggest elevated gradients that exceed both SEA and the modified SEA (T-T, fig. 2) gradients. This supports a range of gradients for eastern Australian Mesozoic-Cenozoic geotherms, going from cooler gradients (e.g., Mesozoic Ruby Hill and Jugiong suites; Sutherland 1999, Gaul et al. 2003) into higher gradients (e.g., Cenozoic western Victoria SEA; O'Reilly et al. 1997, Taylor 1998) and some more extreme gradients (e.g., Cenozoic Tasmania, this study). The exact geotherm gradient becomes critical when correlating other lithological temperatures, uncontrolled by pressure, as for spinel-metalherzolites, garnet-metaclinopyroxenites and non-garnetiferous granulites, leading to potential depth uncertainties in crust-mantle sections (Sutherland et al. 1998).

Some temperature intervals for Table Cape vent assemblages are shown in relation to the TP controlled segments of Tasmanian Miocene geotherms (fig. 2). The spinelmetalherzolite $T$ ranges at Table Cape intersect at up to $1.15 \mathrm{GPa}$ on the modified SEA geotherm, up to $1.0 \mathrm{GPa}$ on the SEA geotherm and extrapolate to $0.8 \mathrm{GPa}$ on a geotherm $80^{\circ} \mathrm{C}$ higher than SEA. Similarly, granulites at Table Cape intersect at up to $1.05 \mathrm{GPa}$ on modified SEA, up to $0.9 \mathrm{GPa}$ on SEA and extrapolate to $0.75 \mathrm{GPa}$ on SEA $+80^{\circ} \mathrm{C}$ geotherms. These potential differences in lithological assignments $(0.3 \mathrm{GPa})$ graphically illustrate their dependence on the precise geotherm, and are further complicated by pressure-dependant variation in metaperidotite T estimates using Witt-Eickschen \& Seck (1991) thermometry.

The seismic data for the Table Cape region suggests a Moho depth at $32 \mathrm{~km}$ (Rawlinson et al. 2001), i.e., 1.05-1.1GPa, which would give $\mathrm{T}$ intersections of $870^{\circ} \mathrm{C}$ on modified SEA, $920^{\circ} \mathrm{C}$ on SEA and $1000^{\circ} \mathrm{C}$ on SEA + $80^{\circ} \mathrm{C}$ geotherms. The first two $\mathrm{T}$ intersections are compatible (within error) with the Table Cape granulite-metalherzolite interval $\left(870-915^{\circ} \mathrm{C}\right)$, but less so for the SEA $\left(+80^{\circ} \mathrm{C}\right)$ geotherm. Thus, an apparent discrepancy exists between more elevated gradients in the lower (grt-metawebsterite) part of the section and reduced gradients at the mantle-crust transition zone. Unless all the thermobarometry (past and present) on Table Cape garnet-metapyroxenites is seriously 
in question, this implies a localised thermal anomaly.

One possibility for such a thermal anomaly is local heating of mantle adjacent to magma chambers. Such mantle magma chambers exist nearby at Boat Harbour, where basanite magmas were fractionated to erupt as lherzolite-bearing nepheline mugearites (Sutherland et al. 1996). The Boat Harbour fractionated lavas are dated at $14.2 \pm 0.1 \mathrm{Ma}$, just prior to eruption of the Table Cape centre (minimum 13.3 \pm 0.2 for late stage lavas). Thus, additionally heated mantle may have already been present when fractionated olivine nephelinite brought up the Table Cape xenolith suite. This scenario would represent small-scale local perturbation of a prevailing geotherm, within a limited segment of the lithosphere. It would contrast with the western Victorian scenario where extensive basaltic intrusions around the mantle-crust boundary were identified on seismic sections and were considered to generate the SEA geotherm (Cull et al. 1991). The Bow Hill TP plots also suggest a declining geothermal gradient, so that thermal contribution from deep mantle magma chambers that fractionated the host nepheline hawaiite there also needs consideration.

\section{SYNTHESIS}

The Table Cape vent xenolith suite (erupted with evolved nephelinite) represents a unique lithospheric section, both within Tasmania and elsewhere among eastern Australian xenolith suites. It is one of two known garnet-bearing (two-pyroxene) xenolith suites in Tasmania, but represents a higher level mantle section with more Fe-rich minerals in the assemblages than occurs at Bow Hill.

Revised thermobarometry for Tasmanian garnet-bearing xenoliths confirmed that the associated Miocene geotherms in part exceeded the western Victorian (SEA) gradient.

Considerations of xenolith temperatures for the crustmantle boundary lithology at Table Cape, in relation to the seismic Moho (32 km depth) and proposed SEA and Table Cape geotherms, suggest the Table Cape geotherm may mark a locally highly perturbed gradient.

The Table Cape geotherm may reflect thermal input from adjacent magma chamber activity that gave $14 \mathrm{Ma}$ Boat Harbour fractionated basalts, prior to Table Cape activity.

Xenolith-derived geotherms related to Mesozoic-Cenozoic basaltic activity have been constructed from many eastern Australian and Asian sites. However, not only do they involve complications in thermobarometry in defining such geotherms, but also incorporate diverse magmatic thermal inputs that may include localised effects.

\section{ACKNOWLEDGEMENTS}

This paper is dedicated to Lance Raynor, whose death in 1987 delayed completion of this detailed study. Additional analyses by F. L. Sutherland were made at the Microscope Unit, University of Sydney and by Dr B. J. Barron, at the Earth Science Laboratories at Macquarie University, Sydney. The new temperature-pressure data for the Bow Hill, Tasmanian xenoliths came from the Taylor 1998 TP program through Dr Ian Roach, MCA lecturer, CR LEME, Department of Earth and Marine Science, Australian National University, Canberra. A 1:25 000 Wynyard geological map was provided by John Everard, Mineral Resources Tasmania, Hobart.
Manuscript compilation was assisted by Sue Folwell, Roger Springthorpe, Ian Graham and Carl Bento, Australian Museum. Funds for field and analytical work were supported by The Australian Museum Trust. The paper was read by Dr I. T. Graham, Geodiversity Research Centre, Australian Museum, Dr L. M. Barron, Geological Survey of New South Wales and Dr B. J. Barron, Sydney. Constructive improvements in the script for publication were made by Dr I Roach, Australian National University and J.L. Everard, Mineral Resources Tasmania.

\section{REFERENCES}

Adam, J. 1990: The geochemistry and experimental petrology of sodic alkaline basalts from Oadlands, Tasmania. Joumal of Petrology 31: 1201-1223.

Ai, Y. 1994: A revision of the garnet-clinopyroxene Fe2+-Mg exchange geothermometer. Contributions to Mineralogy and Petrology 115: 467-473.

Alaoui, H.C., Kornprobst, J. \& Laporte, D. 1997: Inconsistencies between cpx-gnt geothermometry and field observations: example of the pcraluminous eclogites from Beni Bousera (North Morocco). Terra Nova 9(2): 83-86.

Beyer, E.E. 1998: Contrasting characteristics of Proterozoic and Phanerozoic mantle types: preliminary results. 14 th Australian Geological Convention, Townsville: Geological Society of Australia Abstracts 49: 31 .

Beyer, E., O’Reilly, S. \& Zhang, M. 2001: Mantle mixup beneath Tasmania. Research highlights. GEMOC Annual Report 2001: 38.

Brey, G.P. \& Köhler, T. 1990: Geothermobarometry in four phase lherzolites. II New thermometers and practical assessment of existing thermobatometers. Contributions to Mineralogy and Petrology 55: 217-230.

Brown, A.V. 1988: Geology and platinum group-element geochemistry of the Serpentine Hill Complex, Dundas Trough, western Tasmania. Canadian Mineralogist 26: $161-175$.

Cawthorn, R.G. \& Collerson, K.D. 1974: The recalculation of pyroxene end-member parameters and the estimation of ferrous and ferric iron content from electron microprobe analyses. American Mineralogist 59 (11-12): 1203-1208.

Chen, C-Y., Frey, F.A. \& Song, Y. 1989: Evolution of the upper mantle beneath southeast Australia: geochemical evidence from peridotite xenoliths in Mount Leura basanite. Earth and Planetary Science Letters 93: 195-209.

Chen, S., O'Reilly, S.Y., Zhou, X., Griffin, W.L., Zhang, G., Sun, M., Feng, J. \& Zhang, M. 2001: Thermal and petrological structure of the lithosphere beneath Hannuoba, Sino-Korean Craton, China: Evidence from xenoliths. Lithos 56: 267-301.

Cull, J.P., O'Reilly, S.Y. \& Griffin, W.L. 1991: Xenolith geotherms and crustal models in Eastern Australia. Tectonophysics 192: 359-366.

Ellis, D.J. \& Green, D.H. 1979: An experimental study on the effect of $\mathrm{Ca}$ upon garnet-clinopyroxene $\mathrm{Fe}-\mathrm{Mg}$ exchange equilibria. Contributions to Mineralogy and Petrology 71 : $13-22$.

Everard, J.L. 2001: Inclusions of high pressure origin in Tasmanian Cenozoic basalts: A catalogue of localities. Tasmanian Geological Survey Record 2001/9.

Everard, J.L. 2003: Wynyard. Sheet 3846. Digital Geological Atlas 1: 25000 Series. Mineral Resources Tasmania, Hobart.

Frey, F.A., Green, D.H. \& Roy, S.D. 1978: Integrated models of basalt petrogenesis: a study of quartz tholeintes to olivine melilitites from south eastern Australia utilizing geochemical and experimental petrological data. Journal of Petrology 19: 463-513. 
Gaul, O.F., O'Reilly, S.Y. \& Griffin, W.L. 2003: Lithosphere structure and evolution in southeastern Australia. Geological Society of Australia Special Publication 22 and Geological Society of America Special Paper 372: 185-202.

Gautheron, C. \& Moreira, M. 2003: Re-interpretation of the existence of a primitive plume under Australia based on neon isotope fractionation during step heating. Terra Nova 15: 36-39.

Gee, R.D. 1971: Table Cape. Geological Atlas 1 Mile Series. Geological Survey Explanatory Report, Sheet 22 (8016S). Tasmania Department of Mines.

Graham, I.T., Hollis, J.D., Sutherland, F.L. \& Joyce, E.B. 2003: Insights into the Newer Volcanics Province of Victoria. Specialist Group in Geochemistry, Mineralogy and Petrology Field Guide, Geological Society of Australia, Sydney: $110 \mathrm{pp}$.

Griffin, W.L., Sutherland, F.L. \& Hollis, J.D. 1987: Geothermal profile and crust-mantle transition beneath east-central Queensland: volcanology, xenolith petrology and seismic data. Journal of Volcanology and Geothermal Research 31: 177-203.

Griffin, W.L., Wass, S.Y. \& Hollis, J.D. 1984: Ultramafic xenoliths from Bullenmerri and Gnotuk Maars, Victoria, Australia: Petrology of a sub-continental crust-mantle transition. Joumal of Petrology 25: 53-87.

Irving, A.J. 1974: Geochemical and high pressure experimental studies of garnet pyroxenite and pyroxene granulite xenoliths from the Delegate basaltic pipes, Australia. Joumal of Petrology 15: 1-40.

Knowles, C.R. 1987: Garnet end member recasting. Computers and Geosciences 13(6): 656-658.

Le Maitre, R.W. (ed): 1989: A Classification of Igneous Rocks and Glossary of Terms. Blackwell Scientific Publications, Oxford: 193 pp.

McDonough, W.F., Rudnick, R.L. \& McCulloch, M.T. 1991 : The chemical and isotopic composition of the lower eastern Australian lithosphere. Geological Society of Australia Special Publication 17: 163-188.

O'Reilly, S.Y. \& Griffin, W.L. 1985: A xenolith-derived geotherm for southeastern Australia and its geological implications. Tectonophysics 111: 41-63.

O'Reilly, S.Y., Griffin, W.L. \& Gaul, O. 1997: Paleogeothermal gradients in Australia: key to 4-D lithosphere. AGSO Joumal of Australian Geology of Geophysics 17: 63-72.

O'Reilly, S.Y., Nicholls, I.A. \& Griffin W.L. 1989: Xenoliths and megacrysts of eastern Australia, In Johnson, R.W. (ed.). Intraplate Volcanism in Eastern Australia and New Zealand. Cambridge University Press, Cambridge: 254-274.

O'Reilly, S.Y. \& Griffin, W.L. 2000: Apatite in the mantle: implications for metasomatic processes and high heat production in Phanerozoic mantle. Lithos 53: 217-232.

Piestrzeniewicz, R. 1972: Ultramafic inclusions in some Tasmanian basic volcanic rocks. Unpublished B.Sc.(Hons) thesis, University of Tasmania, Hobart.

Rawlinson, N., Houseman, G.A., Collins, C.D.N. \& Drummond, B.J. 2001: New evidence of Tasmania's tectonic history from a novel seismic experiment. Geophysical Research Letters 28(17): 3337-3340.

Rawlinson, N., Reading, A. \& Kennett, B. 2004: Threedimensional structure of the lithosphere beneath northern Tasmania from seismic travel times. 17th Australian Geological Convention, Hobart: Geological Society of Australia Abstracts 73: 113.

Roach, I.C. 2004: Mineralogy, textures and P-T relationships of a suite of xenoliths from the Monaro Volcanic Province, New South Wales, Australia. Joumal of Petrology 45: 1-20.

Sachtleben, T. \& Seck, H.A. 1981: Chemical control of A1solubility in orthopyroxene and its implications on pyroxene geothermometry. Contributions to Mineralogy and Petrology 78: 157-165

Smith, D. 1999: Temperatures and pressures of mineral equilibration in peridotite xenoliths: review, discussion and implications. In Fei, Y., Bertka, C.M. \& Mysen, B.O. (eds): Mantle petrology: Field Observations and high-pressure experimentation. A tribute to Francis $\mathrm{R}$. (Joe) Boyd. Geochemical Society Special Publication 6. The Geochemical Society (Houston): 171-188.

Stolz, A.J. 1984: Garnet websterites and associated ultramafic inclusions from a nepheline mugearite in the Walcha area, New South Wales, Australia. Mineralogical Magazine 48: $167-179$.

Sutherland, F.L. 1999: Volcanism, geotherms, gemstones and lithosphere, since orogenesis, N.E. New South Wales: A Synthesis. In Flood, P.G. (ed): New England Orogen. Earth Sciences, University of New England, Armidale, NSW: 355-364

Sutherland, F.L. \& Wellman, P. 1986: Potassium-argon ages of Tertiary volcanic rocks, Tasmania. Papers and Proceedings of the Royal Society of Tasmania 120: 77--86.

Sutherland, F.L., Hendry, D.F., Barron, B.J., Matthews, W.L. \& Hollis, J.D. 1996: An unusual Tasmanian Tertiary basalt sequence near Boat Harbour, Northwesr Tasmania. Records of the Australian Museum 48: 131-161.

Sutherland, F.L., Graham, I.T., Everard, J.L., Forsyth, S.M. \& Zwingmann, H. 2004: Cenozoic basalts, Tasmania: Landscapes, exposures, ages, petrology, geochemistry, entrainments and petrogenesis. 17th Australian Geological Convention, Hobart: Field Guide A5. Geological Society of Australia, Sydney: $58 \mathrm{pp}$.

Sutherland, F.L., Ewart, A., Raynor, L.R., Hollis, J.D. \& McDonough, W.D. 1989: Tertiary basaltic magmas and the Tasmanian lirhosphere. In Burrett, C.F. \& Martin, E.L. (eds): Geology and Mineral Resources of Tasmania. Geological Society of Australia Special Publication 15: $386-398$

Sutherland, F.L., Hollis, J.D. \& Barron, L.M. 1984: Gannet lherzolite and other inclusions from a basalt flow, Bow Hill, Tasmania. In Kornprobst, J. (ed.): Kimberlites II, The Mantle and Crust-Mantle Relationship. Elsevier, Amsterdam: 145-160.

Sutherland, F.L., Pogson, R.E. \& Barron, B.J. 1998: Discussion. In Discussion and Reply. Palaeogeothermal gradients in Australia: key to 4-D lithospheric mapping. Australian Journal of Earth Sciences 45: 817-821.

Sutherland, F.L., Raynor, L.R. \& Pogson, R.E. 1994: Spinel to garnet therzolite transicion in relation to high temperature palaeogeotherms, eastern Australia. Australian Joumal of Earth Sciences 41: 205-220.

Taylor, W.R. 1998: An experimental test of some geothermometer and geobarometer formulations for upper mantle peridotites with applications to the thermobarometry of fertile therzolite and garnet websterite. Neues Jabrbuch Mineralogie Abhandlugen 172: 381-408.

Tilley, C.E., \& Thompson, R.N. 1972: Melting relations of some ultra alkaline rocks. Geological Journal (Liverpool) 8: $65-70$.

Tribaudino, M. \& Bruno, E. 1993: Effect of Al on enstatite solubility in CMAS clinopyroxenes: 1-Experimental results in the clinopyroxene-orthopyroxene two phase field at $\mathrm{P}=$ 18 kbar. European Journal of Mineralogy 5: 123-131.

Varne, R. 1997: Origin of spinel therzolite inclusions in basalric rocks from Tasmania and elsewhere. Journal of Petrology 18: $1-23$

Wells, P.R.A. 1977: Pyroxene thermometry in simple and complex systems. Contributions to Mineralogy and Petrology 62 : 129-139.

Wilkinson, J.F.G. \& Stolz A.J. 1997: Subcalcic clinopyroxenites and associated ultramafic xenoliths in alkali basalt near Glen Innes, northeastern New South Wales, Australia. Contributions to Mineralogy and Petrology 127: 272-290.

Wilshire, H.G. \& Shervais, J.W. 1975: Al-augite and Crdiopside ultramafic xenoliths in basaltic rocks from the 
western United States. Physics and Chemistry of the Earth 9: $257-272$.

Witt-Eickschen, G. \& Seck, H.A. 1991: Solubility of Ca and $\mathrm{Al}$ in orthopyroxene from spinel peridotite: an improved version of an empirical geothermometer. Contributions to Mineralogy and Petrology 106: 431-439.

Wood, B.J. 1974: Solubility of alumina in orthopyroxene coexisting with garnet. Contributions to Mineralogy and Petrology 46: 1-15.
Xu, X., O'Reilly, S.Y., Griffin, W.L., Zhou, X. \& Huang, X. 1998: The nature of the Cenozoic lithosphere at Nushan, Eastern China. In Flower, M.F.J., Chung, S.L., Lo, C.H. \& Lee, Y.Y. (eds): Mantle dynamics and plate interactions in East Asia. Geodynamics Series 27, American Geophysical Union, Washington, DC: 167--196.

(accepted 29 November 2004) 